\title{
Gauge dependence and multiplicative renormalization of Yang-Mills theory with matter fields
}

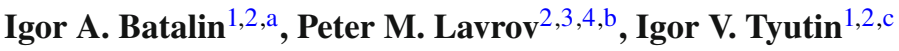 \\ ${ }^{1}$ Physical Institute, Leninsky Prospect 53, 119991 Moscow, Russia \\ 2 Tomsk State Pedagogical University, Kievskaya St. 60, 634061 Tomsk, Russia \\ ${ }^{3}$ National Research Tomsk State University, Lenin Av. 36, 634050 Tomsk, Russia \\ ${ }^{4}$ Departamento de Física, ICE, Universidade Federal de Juiz de Fora, Campus Universitário-Juiz de Fora, Juiz de Fora, MG 36036-900, Brazil
}

Received: 4 March 2019 / Accepted: 16 July 2019 / Published online: 26 July 2019

(C) The Author(s) 2019

\begin{abstract}
In the paper, within the background field method, the renormalization and the gauge dependence is studied as for an SU(2) Yang-Mills theory with multiplets of spinor and scalar fields. By extending the quantum action of the BVformalism with an extra fermion vector field and a constant fermion parameter, the multiplicative character of the renormalizability is proven. The renormalization of all the physical parameters of the theory under consideration is shown to be gauge-independent.
\end{abstract}

\section{Introduction}

When constructing modern models of fundamental interactions [1], non-Abelian gauge field theories [2] play a central role. Any gauge theory can be quantized in a covariant way within the BV-formalism $[3,4]$ involving the gauge-fixing procedure as an important tool. Although at the quantum level the gauge symmetry is broken, nevertheless, it causes the existence of fundamental global supersymmetry known as the BRST symmetry [5,6]; application of the BRST symmetry to renormalization of gauge theories has been proposed in paper [7]. The respective conserved fermion nilpotent generator is known as the BRST charge $[3,4]$ responsible for correct construction of physical state space $[8,9]$. Due to the equivalence theorem [10] and the BRST symmetry, it is succeeded to prove that the physical $S$-matrix is independent of the choice of gauge fixing.

The gauge dependence problem did appear by itself from the study of the effective potential, which appeared to be gauge-dependent in Yang-Mills theories with the spon-

\footnotetext{
a e-mail: batalin@1pi.ru

be-mail: lavrov@tspu.edu.ru

c e-mail: tyutin@lpi.ru
}

taneous symmetry breaking, when calculating physicallysensible results (the energy of the ground state, the masses of the physical particles, and so on) $[11,12]$. In Refs. $[13,14]$ it was established that the energy of the ground state was gaugeindependent. Later, it was proved $[15,16]$ that in Yang -Mills theories the dependence of gauge parameters in the effective action could be described in terms of gauge-invariant functional whose arguments (fields) were gauge-dependent (see also recent Refs. $[19,20]$ devoted to that problem as resolved via the procedure of redefinition of the field variables, found in $[15,16])$. When studying the gauge dependence problem it was a nice idea as to enlarge the usual BRST transformations by transforming also the gauge parameter $\xi$ into fermion variable $\chi$, which results in the extension of the master equation [17]. We have used a similar extension in the present paper, together with introducing new fermion fields $\theta_{\mu}^{a}$ [18].

Our investigation of renormalization and gauge dependence in an SU(2) Yang-Mills theory with spinor and scalar fields is based on using the background field formalism $[21,22,24]$. This formalism is the popular method for quantum studies and calculations in gauge theories because it allows one to work with the effective action invariant under the gauge transformations of the background fields, and to reproduce all usual physical results which can be obtained within the standard quantization approach. Various aspects of quantum properties of Yang-Mills theories and quantum gravity theories have been successfully studied in this technique [18,25-31] (among recent applications see, for example, [32-35]).

Although there are many papers devoted to various aspects of renormalizability of Yang-Mills theories, the gauge dependence of the renormalization constants has been studied explicitly only as for the gauge field sector [18]. In recent paper [34], we have studied a structure of the renormalization procedure and a gauge dependence as for an Yang- 
Mills theory with a multiplet of spinor fields based on an arbitrary simple compact gauge Lie group. We have proved the multiplicativity of the renormalization and the gauge independence of the renormalization constants of the physical parameters of the theory (gauge interaction constant and fermion mass parameter). The main goal of the present paper is to include scalar fields into a gauge model as to have the possibility of generating masses to physical particles through the spontaneous symmetry breaking [36]. Thereby, we have generalized the previous results to the maximal spectrum of spins, $\{0,1 / 2,1\}$, capable to meet the requirement of multiplicative renormalizability. In contrast to the case of spinor fields, the multiplicative renormalizability in the sector of scalar fields is not provided with the gauge symmetry only, and this requires for an additional proof. Also, specific problems may appear when studying the gauge independence of renormalization constants of new couplings of scalar fields which do not connect directly with Yang-Mills interactions. Thus, the full description of the renormalization structure requires for a detailed explicit analysis of the respective coupled equations, which is presented in the paper. To simplify the presentation and calculations, we restrict ourselves by the simplest Yang-Mills theory with SU(2) gauge group. Generalization of our results to another gauge group can be made straightly.

The paper is organized as follows. In Sect. 2, the action of SU(2) Yang-Mills theory with multiplets of spinor and scalar fields in the background field method is extended with the help of additional fermion vector field and constant fermion parameter which allows one later to arrive at a multiplicative renormalizable theory. The symmetries of this extended action are studied and presented in a set of equations for that action. In Sect. 3, it is established the structure and the arbitrariness is described for any local functional with the quantum numbers of the extended action that satisfies the same set of equations as the extended action does. In Sect. 4, the equations are derived for the generating functional of vertexes (effective action), as a consequence at the quantum level, of the symmetry property of the extended action; and it is shown that the generating functional of vertexes satisfies the same equations as the extended quantum action does. In Sect. 5, it is studied the renormalization procedure of the theory considered when using the loop expansion technique and the minimal subtraction scheme; and thus the multiplicative renormalizability of the theory is proved. In Sect. 6, the gauge independence of all physical parameters of the theory under consideration to any order of loop expansions is found. Concluding remarks are given in Sect. 7.

Condensed DeWitt's notations [37] are used through the paper. Functional derivatives with respect to field variables are understood as the left. Right derivatives of a quantity $f$ with respect to the variable $\varphi$ are denoted as $f \frac{\overleftarrow{\delta}}{\delta \varphi}$

\section{Extended quantum action}

Let us consider an $S U$ (2)-gauge theory of non-Abelian vector fields $A_{\mu}^{\alpha}=A_{\mu}^{\alpha}(x)$, a doublet of spinor fields $\psi_{j}=$ $\psi_{j}(x), \bar{\psi}_{j}=\bar{\psi}_{j}(x)$ and a triplet of real scalar fields $\varphi^{\alpha}=\varphi^{\alpha}(x)$ in the $\mathrm{d}=4$ Minkowski space-time, with the action

$$
\begin{gathered}
\mathcal{S}_{Y M}(A, \Psi, \varphi)=\mathcal{S}_{1}(A)+\mathcal{S}_{2}(A, \Psi)+\mathcal{S}_{3}(A, \varphi) \\
+\mathcal{S}_{4}(\Psi)+\mathcal{S}_{5}(\varphi)+\mathcal{S}_{6}(\Psi, \varphi), \\
\mathcal{S}_{1}(A)=-\frac{1}{4} \int d x G_{\mu \nu}^{\alpha}(A) G_{\mu \nu}^{\alpha}(A), \\
\mathcal{S}_{2}(A, \Psi)=\int d x i \bar{\psi}_{j} \gamma^{\mu} D_{\psi \mu j k}(A) \psi_{k}, \\
\mathcal{S}_{3}(A, \varphi)=\frac{1}{2} \int d x D_{\varphi \mu}^{\alpha \beta}(A) \varphi^{\beta} D_{\varphi \mu}^{\alpha \gamma}(A) \varphi^{\gamma}, \\
\mathcal{S}_{4}(\Psi)=-m \int d x \bar{\psi}_{j} \psi_{j}, \\
\mathcal{S}_{5}(\varphi)=\int d x\left(-\frac{M^{2}}{2} \varphi^{2}-\frac{\lambda}{4} \varphi^{4}\right), \\
\mathcal{S}_{6}(\Psi, \varphi)=i \vartheta \int d x \varphi^{\alpha} \bar{\psi}_{j} t_{j k}^{\alpha} \psi_{k},
\end{gathered}
$$

where the notations

$$
\begin{aligned}
& G_{\mu \nu}^{\alpha}(A)=\partial_{\mu} A_{v}^{\alpha}-\partial_{\nu} A_{\mu}^{\alpha}+g \varepsilon^{\alpha \beta \gamma} A_{\mu}^{\beta} A_{v}^{\gamma}, \\
& D_{\psi \mu j k}(A)=\delta_{j k} \partial_{\mu}+g t_{j k}^{\alpha} A_{\mu}^{\alpha}, \quad t_{j k}^{\alpha}=\frac{i}{2} \sigma_{j k}^{\alpha}, \\
& \Psi=\{\psi, \bar{\psi}\}, \\
& D_{\varphi \mu}^{\alpha \beta}(A)=\delta_{\alpha \beta} \partial_{\mu}+g E_{\alpha \beta}^{\gamma} A_{\mu}^{\gamma}, \\
& \varphi^{2}=\varphi^{\alpha} \varphi^{\alpha}, \quad \varphi^{4}=\left(\varphi^{2}\right)^{2}, \\
& E_{\alpha \beta}^{\gamma}=\varepsilon^{\alpha \gamma \beta}, \quad \alpha, \gamma, \beta=1,2,3, \quad j, k=1,2,
\end{aligned}
$$

were used. In the relations (2.1)-(2.4) and (2.5) $\varepsilon^{\alpha \beta \gamma}$ represent the structure coefficients of the $S U$ (2) gauge group, $t^{\alpha}=\left\{t_{j k}^{\alpha}\right\}$ and $E^{\alpha}=\left\{E_{\beta \gamma}^{\alpha}\right\}$ are the generators of gauge transformations of spinor and scalar fields with the properties

$$
\begin{aligned}
{\left[E^{\alpha}, E^{\beta}\right] } & =\varepsilon^{\alpha \beta \gamma} E^{\gamma}, \quad\left[t^{\alpha}, t^{\beta}\right]=\varepsilon^{\alpha \beta \gamma} t^{\gamma}, \\
\left(t^{\gamma}\right)^{+} & =-t^{\gamma}, \quad\left[\gamma^{\mu}, t^{\alpha}\right]=0,
\end{aligned}
$$

$\gamma^{\mu}$ are the Dirac matrices, $\sigma_{j k}^{\alpha}$ are the Pauli matrices and $g$ is a gauge coupling parameter, $\lambda$ is a coupling constant of the scalar field, $\vartheta$ is a coupling constant of the scalar and spinor fields, $m$ and $M$ are mass parameters of the spinor and the scalar fields, respectively. 
The action (2.1) is invariant under the $S U(2)$-gauge transformations with gauge parameters $\omega_{\alpha}=\omega_{\alpha}(x)$,

$\delta_{\omega} \mathcal{S}_{Y M}(A, \Psi, \varphi)=0$

$\delta_{\omega} A_{\mu}^{\alpha}=\left(\delta_{\alpha \beta} \partial_{\mu}+g \varepsilon^{\alpha \sigma \beta} A_{\mu}^{\sigma}\right) \omega_{\beta}=D_{\mu}^{\alpha \beta}(A) \omega_{\beta}$,

$\delta_{\omega} \psi_{j}=-g t_{j k}^{\beta} \psi_{k} \omega_{\beta}, \quad \delta_{\omega} \bar{\psi}_{j}=g \bar{\psi}_{k} t_{k j}^{\beta} \omega_{\beta}$,

$\delta_{\omega} \varphi^{\alpha}=-g E_{\alpha \gamma}^{\beta} \varphi^{\gamma} \omega_{\beta}$.

Notice that the form (2.4) of the polynomial $\mathcal{S}_{5}(\varphi)$ is uniquely determined by the invariance requirement under global $S U(2)$-transformations.

In the background-field formalism [21,22,24] the gauge field $A_{\mu}^{\alpha}$ appearing in the classical action (2.1) is replaced by $A_{\mu}^{\alpha}+\mathcal{B}_{\mu}^{\alpha}$,

$\mathcal{S}_{Y M}(A, \Psi, \varphi) \rightarrow \mathcal{S}_{Y M}(A+\mathcal{B}, \Psi, \varphi)$,

where $\mathcal{B}_{\mu}^{\alpha}$ is considered as an external vector field. Effective action for functional integral in quantum theory is constructed with using the BV-formalism $[3,4]$.

To study a renormalization structure and a gauge dependence of renormalization constants we use an extended action. This action $S_{\text {ext }}=S_{\text {ext }}\left(Q, Q^{*}, \bar{C}, B, \mathcal{B}, \xi, \theta, \chi\right)$ is constructed by introducing additional fermion fields $\theta_{\mu}^{\alpha}=$ $\theta_{\mu}^{\alpha}(x)$ and a constant fermion parameter ${ }^{1} \chi$, and has the form

$$
\begin{aligned}
S_{\text {ext }}= & \mathcal{S}_{Y M}(A+\mathcal{B}, \Psi, \varphi)+\int d x Q^{*} \mathcal{R}_{Q} \\
& +\int d x\left(\bar{C}^{\alpha} D_{\mu}^{\alpha \gamma}(\mathcal{B}) D_{\mu}^{\gamma \beta}(A+\mathcal{B}) C^{\beta}\right. \\
& \left.+B^{\alpha} D_{\mu}^{\alpha \beta}(\mathcal{B}) A_{\mu}^{\beta}+(\xi / 2) B^{\alpha} B^{\alpha}\right) \\
& +\int d x\left(\theta_{\mu}^{\alpha}\left[D_{\mu}^{\alpha \beta}(A+\mathcal{B}) \bar{C}^{\beta}-A_{\mu}^{* \alpha}\right]\right. \\
& +\chi\left[\left(A_{\mu}^{* \alpha}-D_{\mu}^{\alpha \beta}(\mathcal{B}) \bar{C}^{\beta}\right) A_{\mu}^{\alpha}\right. \\
& \left.\left.+C^{* \alpha} C^{\alpha}+\psi_{j}^{*} \psi_{j}+\bar{\psi}_{j}^{*} \bar{\psi}_{j}+\varphi^{* \alpha} \varphi^{\alpha}\right]\right),
\end{aligned}
$$

where $Q$ means the set of fields $\left\{A_{\mu}^{\alpha}, \psi_{j}, \bar{\psi}_{j}, \varphi^{\alpha}, C^{\alpha}\right\}$, the symbol $Q^{*}$ is used for the set of corresponding antifields and $\mathcal{R}_{Q}$ are generators of the BRST transformations [5,6],

$\mathcal{R}_{A_{\mu}^{\alpha}}=D_{\mu}^{\alpha \beta}(A+\mathcal{B}) C^{\beta}, \quad \mathcal{R}_{\psi_{j}}=-g t_{j k}^{\alpha} \psi_{k} C^{\alpha}$,

$$
\mathcal{R}_{\bar{\psi}_{j}}=g \bar{\psi}_{k} t_{k j}^{\alpha} C^{\alpha} \text {, }
$$

$\mathcal{R}_{\varphi^{\alpha}}=-g E_{\alpha \gamma}^{\beta} \varphi^{\gamma} C^{\beta}, \quad \mathcal{R}_{C^{\alpha}}=\frac{g}{2} \varepsilon^{\alpha \beta \gamma} C^{\beta} C^{\gamma}$.

The action (2.9) is invariant,

$S_{\text {ext }} \frac{\overleftarrow{\delta}}{\delta \Pi_{I}} \delta \Pi_{I} \epsilon=0$

${ }^{1}$ For the first time such additional variables were used in [18]. under the following global supersymmetry transformations of the variables (here $\left\{\Pi_{I}\right\}$ is the set of all the variables entering the action, $\epsilon$ is a constant fermion parameter of the transformation, $\left.\varepsilon(\epsilon)=1, \epsilon^{2}=0\right)$ ),

$$
\begin{aligned}
& \delta A_{\mu}^{\alpha}=D_{\mu}^{\alpha \beta}(A+\mathcal{B}) C^{\beta}+\theta_{\mu}^{\alpha}=\frac{\delta}{\delta A_{\mu}^{* \alpha}} S_{\mathrm{ext}}+\chi A_{\mu}^{\alpha}, \\
& \delta B^{\alpha}=-\frac{1}{\xi}\left[D_{\mu}^{\alpha \gamma}(\mathcal{B}) D_{\mu}^{\gamma \beta}(A+\mathcal{B}) C^{\beta}\right. \\
& \left.+D_{\mu}^{\alpha \beta}(A+\mathcal{B}) \theta_{\mu}^{\beta}+\chi D_{\mu}^{\alpha \beta}(\mathcal{B}) A_{\mu}^{\beta}\right]-\chi B^{\alpha} \\
& =-\frac{1}{\xi} \frac{\delta}{\delta \bar{C}^{\alpha}} S_{\mathrm{ext}}-\chi B^{\alpha}, \\
& \delta C^{\alpha}=\frac{g}{2} \varepsilon^{\alpha \beta \gamma} C^{\beta} C^{\gamma}=\frac{\delta}{\delta C^{* \alpha}} S_{\mathrm{ext}}-\chi C^{\alpha}, \\
& \delta \bar{C}^{\alpha}=-\frac{1}{\xi} D_{\mu}^{\alpha \beta}(\mathcal{B}) A_{\mu}^{\beta}+\chi \bar{C}^{\alpha} \\
& =-\frac{1}{\xi} \frac{\delta}{\delta B^{\alpha}} S_{\mathrm{ext}}+B^{\alpha}+\chi \bar{C}^{\alpha}, \\
& \delta \psi_{j}=-g t_{j k}^{\alpha} \psi_{k} C^{\alpha}=\frac{\delta}{\delta \psi_{j}^{*}} S_{\mathrm{ext}}-\chi \psi_{j}, \\
& \delta \bar{\psi}_{j}=g \bar{\psi}_{k} t_{k j}^{\alpha} C^{\alpha}=\frac{\delta}{\delta \bar{\psi}_{j}^{*}} S_{\mathrm{ext}}-\chi \bar{\psi}_{j}, \\
& \delta \varphi^{\alpha}=-g E_{\alpha \gamma}^{\beta} \varphi^{\gamma} C^{\beta}=\frac{\delta}{\delta \varphi^{* \alpha}} S_{\mathrm{ext}}+\chi \varphi^{\alpha}, \\
& \delta A_{\mu}^{* \alpha}=\chi A_{\mu}^{* \alpha}, \delta C^{* \alpha}=-\chi C^{* \alpha}, \delta \psi_{j}^{*}= \\
& -\chi \psi_{j}^{*}, \quad \delta \bar{\psi}_{j}^{*}=-\chi \bar{\psi}_{j}^{*}, \delta \varphi^{* \alpha}=\chi \varphi^{* \alpha}, \\
& \delta \xi=2 \xi \chi, \quad \delta \mathcal{B}_{\mu}^{\alpha}=-\theta_{\mu}^{\alpha}, \quad \delta \theta_{\mu}^{\alpha}=0, \quad \delta \chi=0 \text {. }
\end{aligned}
$$

Taking into account the right-hand sides of the relations (2.11)-(2.14) and omitting indices of all variables, the invariance condition of the action $S_{\text {ext }}$ rewrites in the form of the following equation

$$
\begin{aligned}
& \int d x\left(S_{\mathrm{ext}} \frac{\overleftarrow{\delta}}{\delta Q} \frac{\delta}{\delta Q^{*}} S_{\mathrm{ext}}-B \frac{\delta}{\delta \bar{C}} S_{\mathrm{ext}}-\theta \frac{\delta}{\delta \mathcal{B}} S_{\mathrm{ext}}\right) \\
& +\chi \int d x\left[Q \frac{\delta}{\delta Q}-Q^{*} \frac{\delta}{\delta Q^{*}}-\bar{C} \frac{\delta}{\delta \bar{C}}-B \frac{\delta}{\delta B}\right] S_{\mathrm{ext}} \\
& +2 \chi \xi \frac{\partial}{\partial \xi} S_{\mathrm{ext}}=0
\end{aligned}
$$

Also, the action (2.9) satisfies the equation

$S_{\mathrm{ext}} \overleftarrow{H^{\alpha}} \omega_{\alpha}=0$ 
where

$$
\begin{aligned}
\overleftarrow{H^{\alpha}} \omega_{\alpha}= & \int d x\left\{\left[\frac{\overleftarrow{\delta}}{\delta \mathcal{B}_{\mu}^{\beta}} D_{\mu}^{\beta \alpha}(\mathcal{B})+g \varepsilon^{\beta \gamma \alpha}\right.\right. \\
& \times\left(\frac{\overleftarrow{\delta}}{\delta A_{\mu}^{\beta}} A_{\mu}^{\gamma}+\frac{\overleftarrow{\delta}}{\delta B^{\beta}} B^{\gamma}+\frac{\overleftarrow{\delta}}{\delta C^{\beta}} C^{\gamma}+\frac{\overleftarrow{\delta}}{\delta \bar{C}^{\beta}} \bar{C}^{\gamma}\right) \\
& -g E_{\beta \gamma}^{\alpha}\left(\frac{\overleftarrow{\delta}}{\delta \varphi^{\beta}} \varphi^{\gamma}+\frac{\overleftarrow{\delta}}{\delta \varphi^{* \beta}} \varphi^{* \gamma}\right) \\
& -g t_{j k}^{\alpha}\left(\frac{\overleftarrow{\delta}}{\delta \psi_{j}} \psi_{k}+\frac{\overleftarrow{\delta}}{\delta \bar{\psi}_{j}^{*}} \bar{\psi}_{k}^{*}\right) \\
& +g\left(\frac{\overleftarrow{\delta}}{\delta \bar{\psi}_{j}}+\frac{\overleftarrow{\delta}}{\delta \psi_{j}^{*}} \psi_{k}^{*}\right) t_{k j}^{\alpha} \\
& \left.\left.+g \varepsilon^{\beta \gamma \alpha}\left(\frac{\overleftarrow{\delta}}{\delta A_{\mu}^{* \beta}} A_{\mu}^{* \gamma}+\frac{\overleftarrow{\delta}}{\delta C^{* \beta}} C^{* \gamma}+\frac{\overleftarrow{\delta}}{\delta \theta_{\mu}^{\beta}} \theta_{\mu}^{\gamma}\right)\right] \omega_{\alpha}\right\}
\end{aligned}
$$

is the operator of the gauge transformations in the sector of the fields $\mathcal{B}_{\mu}, \varphi, \psi, \bar{\psi}$ and, at the same time, of the tensor transformations in the sector of the fields $A_{\mu}, C, \bar{C}, B, \theta_{\mu}$, $A_{\mu}^{*}, \varphi^{*}, \psi^{*}, \bar{\psi}^{*}, C^{*}$.

Finally, let us notice the two important relations linear in the fields $A_{\mu}, B$ and their derivatives, which the action (2.9) satisfies to,

$$
\begin{aligned}
& \frac{\delta}{\delta B^{\alpha}} S_{\mathrm{ext}}=D_{\mu}^{\alpha \beta}(\mathcal{B}) A_{\mu}^{\beta}+\xi B^{\alpha}, \\
& D_{\mu}^{\alpha \beta}(\mathcal{B}) \frac{\delta}{\delta A_{\mu}^{* \beta}} S_{\mathrm{ext}}-\frac{\delta}{\delta \bar{C}^{\alpha}} S_{\mathrm{ext}}=-g \varepsilon^{\alpha \beta \gamma} A_{\mu}^{\beta} \theta_{\mu}^{\gamma} .
\end{aligned}
$$

The equation (2.24) means that the action $S_{\text {ext }}$ (2.9) depends on the variables $A_{\mu}^{* \alpha}$ and $\bar{C}^{\alpha}$ only in combination $A_{\mu}^{* \alpha}-$ $D_{\mu}^{\alpha \beta}(\mathcal{B}) \bar{C}^{\beta}$ when $\theta_{\mu}^{\beta}=0$.

The action $S_{\text {ext }}$ can be represented in another useful form,

$S_{\text {ext }}=\Gamma_{0 \mid 0}+\sum_{k=6}^{22} \Gamma_{0 \mid k}+\chi \sum_{k=1}^{5} \Gamma_{0 \mid k}$,

where

$$
\begin{aligned}
& \Gamma_{0 \mid 0}=\int d x\left(B^{\alpha} D_{\mu}^{\alpha \beta}(\mathcal{B}) A_{\mu}^{\beta}+\frac{\xi}{2} B^{\alpha} B^{\alpha}+g \theta_{\mu}^{\alpha} \varepsilon^{\alpha \beta \gamma} A_{\mu}^{\beta} \bar{C}^{\gamma}\right), \\
& \Gamma_{0 \mid 1}=\int d x\left[\mathcal{A}_{\mu}^{* \alpha} \mathcal{A}_{\mu}^{\alpha}\right], \\
& \Gamma_{0 \mid 2}=\int d x\left[C^{* \alpha} C^{\alpha}\right], \Gamma_{0 \mid 3}=\int d x\left[\psi_{j}^{*} \psi_{j}\right] \\
& \Gamma_{0 \mid 4}=\int d x\left[\bar{\psi}_{j}^{*} \bar{\psi}_{j}\right], \Gamma_{0 \mid 5}=\int d x\left[\varphi^{* \alpha} \varphi^{\alpha}\right] \\
& \Gamma_{0 \mid 6}=\int d x\left[\mathcal{A}_{\mu}^{* \alpha} \theta_{\mu}^{\alpha}\right], \\
& \Gamma_{0 \mid 7}=\int d x\left[\mathcal{A}_{\mu}^{* \gamma} D_{\mu}^{\gamma \sigma}(\mathcal{B}) C^{\sigma}\right],
\end{aligned}
$$

$$
\begin{gathered}
\Gamma_{0 \mid 8}=\int d x\left[g \mathcal{A}_{\mu}^{* \gamma} \varepsilon^{\gamma \alpha \sigma} \mathcal{A}_{\mu}^{\alpha} C^{\sigma}\right], \\
\Gamma_{0 \mid 9}=\int d x\left[\frac{g}{2} C^{* \alpha} \varepsilon^{\alpha \beta \gamma} C^{\beta} C^{\gamma}\right], \\
\Gamma_{0 \mid 10}=-\int d x\left[g \psi^{*} t^{\alpha} \psi C^{\alpha}\right], \\
\Gamma_{0 \mid 11}=\int d x\left[g \bar{\psi} t^{\alpha} \bar{\psi}^{*} C^{\alpha}\right], \\
\Gamma_{0 \mid 12}=-\int d x\left[g \varphi^{* \gamma} \varepsilon^{\gamma \alpha \sigma} \varphi^{\sigma} C^{\alpha}\right], \\
\Gamma_{0 \mid 13}=i \int d x\left[\bar{\psi} \gamma^{\mu} D_{\psi \mu}(\mathcal{B}) \psi\right], \\
\Gamma_{0 \mid 14}=i g \int d x\left[\bar{\psi} \gamma^{\mu} t^{\alpha} \mathcal{A}_{\mu}^{\alpha} \psi\right], \\
\Gamma_{0 \mid 15}=-m \int d x[\bar{\psi} \psi], \\
\Gamma_{0 \mid 16}=\int d x\left[\frac{1}{2}\left(D_{\varphi \mu}^{\alpha \beta}(\mathcal{B}) \varphi^{\beta}\right) D_{\varphi \mu}^{\alpha \gamma}(\mathcal{B}) \varphi^{\gamma}\right], \\
\Gamma_{0 \mid 17}=\int d x\left[g \varepsilon^{\alpha \gamma \sigma}\left(D_{\varphi \mu}^{\alpha \beta}(\mathcal{B}) \varphi^{\beta}\right) \mathcal{A}_{\mu}^{\gamma} \varphi^{\sigma}\right], \\
\Gamma_{0 \mid 18}=\int d x\left[\frac{g^{2}}{2} E_{\alpha \beta}^{\rho} \mathcal{A}_{\mu}^{\alpha} \varphi^{\beta} E_{\gamma \sigma}^{\rho} \mathcal{A}_{\mu}^{\gamma} \varphi^{\sigma}\right], \\
\Gamma_{0 \mid 19}=-\frac{M^{2}}{2} \int d x \varphi^{2}, \Gamma_{0 \mid 20}=-\frac{\lambda}{4} \int d x \varphi^{4}, \\
\Gamma_{0 \mid 21}=i \vartheta \int d x\left[\varphi^{\alpha} \bar{\psi} t^{\alpha} \psi\right], \\
\Gamma_{0 \mid 22}=-\frac{1}{4} \int d x\left[G_{\mu \nu}^{\alpha}(\mathcal{A}+\mathcal{B}) G_{\mu \nu}^{\alpha}(\mathcal{A}+\mathcal{B})\right],
\end{gathered}
$$

and the notations

$$
\mathcal{A}_{\mu}^{* \alpha}=A_{\mu}^{* \alpha}-D_{\mu}^{\alpha \beta}(\mathcal{B}) \bar{C}^{\beta}, \quad \mathcal{A}_{\mu}^{\alpha}=A_{\mu}^{\alpha}
$$

are used. Note that the functionals $\Gamma_{0 \mid 0}-\Gamma_{0 \mid 21}$ are homogeneous with respect to fields $\Omega$ and antifields $\Omega^{*}$ while the functional $\Gamma_{0 \mid 22}$ does not obey this property and in symbolic notation has the form

$\Gamma_{0 \mid 22}=\sum_{l=0}^{4} \Gamma_{0 \mid 22, l}, \quad \Gamma_{0 \mid 22, l}=\int d x\left[T_{l}(\mathcal{B}) \mathcal{A}^{l}\right]$,

where $\Gamma_{0 \mid 22, l}$ is homogeneous functional with respect to variable $\mathcal{A}$ of order $l$ and $T_{l}(\mathcal{B})$ is a tensor (differential operator) with $l$ gauge and $l$ Lorentz indices. In what follow we do not need in explicit representation of quantities $T_{l}(\mathcal{B})$.

Later on, we will see that the generating functional of vertex functions (effective action), counterterms and renormalized action satisfy the same Eqs. (2.20), (2.21), (2.22), (2.23), (2.24), as the action $S_{\text {ext }}$ does. Moreover, the counterterms and the renormalized action are linear combinations of the same vertices as the action $S_{\text {ext }}$ is. Explicit form of these vertices will be given in the next section.

Now we give the table of "quantum" numbers of fields, antifields, auxiliary fields and constant parameters used in construction of $S_{\text {ext }}$ : 


\begin{tabular}{lllllllllllllll}
\hline Quantity & $A, \mathcal{B}$ & $\psi, \bar{\psi}$ & $\varphi$, & $C, \bar{C}$ & $B$ & $\xi$ & $\theta$ & $A^{*}$ & $\psi^{*}, \bar{\psi}^{*}$ & $\varphi^{*}$ & $C^{*}$ & $d x\left(\equiv d^{4} x\right)$ & $\partial_{x}$ & $\chi$ \\
\hline$\varepsilon$ & 0 & 1 & 0 & 1 & 0 & 0 & 1 & 1 & 0 & 1 & 0 & 0 & 0 & 1 \\
$\operatorname{gh}$ & 0 & 0 & 0 & $1,-1$ & 0 & 0 & 1 & -1 & -1 & -1 & -2 & 0 & 0 \\
$\operatorname{dim}$ & 1 & $3 / 2$ & 1 & 1 & 2 & 0 & 2 & 2 & $3 / 2$ & 2 & 2 & -4 & 1 \\
$\varepsilon_{f}$ & 0 & $1,-1$ & 0 & 0 & 0 & 0 & 0 & 0 & $-1,1$ & 0 & 0 & 0 & 1 \\
\hline
\end{tabular}

where " $\varepsilon^{\prime \prime}$ describes the Grassmann parity, the symbol " $\mathrm{gh}^{\prime \prime}$ is used to denote the ghost number, "dim" means the canonical dimension and " $\varepsilon_{f}^{\prime \prime}$ is the fermionic number. Using the table of "quantum" numbers it is easy to establish the quantum numbers of any quantities met in the text.

\section{General structure of renormalized action}

It will be shown below that the renormalized action is a local functional of field variables with quantum numbers of the action $\mathcal{S}_{\text {ext }}$ (2.9), which satisfies the same Eqs. (2.20)-(2.24) as the action $S_{\text {ext }}$ does. In this section, we find a general solution to the Eqs. (2.20)-(2.24) under the conditions pointed out above.

So, let $P$ be a functional of the form

$P=\int d x P(x)$

where $P(x)$ is a local polynomial of all variables $Q, Q^{*}, \bar{C}$, $B, \mathcal{B}, \xi, \theta, \chi$ with $\operatorname{dim}(P(x))=4$. Let the functional $P$ satisfies the Eqs. (2.20)-(2.24) (with the substitution $S_{\text {ext }} \rightarrow$ $P)$, and we represent it in the form

$P=\Gamma_{0 \mid 0}+P^{(1)}+\chi P^{(2)}$,

where the functional $\Gamma_{0 \mid 0}$ is defined in (2.26), the functionals $P^{(1)}, P^{(2)}$ do not depend on $\chi$ and obey the properties

$\varepsilon\left(P^{(1)}\right)=0, \quad \operatorname{gh}\left(P^{(1)}\right)=0$,

$\operatorname{dim}\left(P^{(1)}\right)=0, \quad \varepsilon_{f}\left(\left(P^{(1)}\right)\right)=0$,

$\varepsilon\left(P^{(2)}\right)=1, \quad \operatorname{gh}\left(P^{(2)}\right)=-1$,

$\operatorname{dim}\left(P^{(2)}\right)=-1, \quad \varepsilon_{f}\left(\left(P^{(2)}\right)\right)=0$.

From the Eq. (2.23) for $P$ and the presentation (3.2) it follows that $P^{(1)}$ and $P^{(2)}$ do not depend on fields $B^{\alpha}$,

$P^{(k)}=P^{(k)}\left(Q, Q^{*}, \bar{C}, \mathcal{B}, \xi, \theta\right), k=1,2$,

With the help of new variables $\mathcal{A}_{\mu}^{* \alpha}(x), \quad \mathcal{A}^{\alpha}{ }_{\mu}$ (2.37), we define new functionals $\tilde{P}^{(k)}$ by the rule

$$
\begin{aligned}
\tilde{P}^{(k)} & =\tilde{P}^{(k)}\left(\Omega, \Omega^{*}, \mathcal{B}, \bar{C}, \xi, \theta\right) \\
& =\left.P^{(k)}\left(Q, Q^{*}, \mathcal{B}, \bar{C}, \xi, \theta\right)\right|_{A^{*} \rightarrow \mathcal{A}^{*}+D(\mathcal{B}) \bar{C}}, \quad k=1,2,
\end{aligned}
$$

and find that $\tilde{P}^{(k)}$ do not depend on fields $\bar{C}^{\alpha}$,

$\tilde{P}^{(k)}=\tilde{P}^{(k)}\left(\Omega, \Omega^{*}, \mathcal{B}, \xi, \theta\right)$.
Omitting the indices of all variables in relations (3.6) and (3.7), the following notations

$\Omega=\{\mathcal{A}, \psi, \bar{\psi}, \varphi, C\}, \quad \Omega^{*}=\left\{\mathcal{A}^{*}, \psi^{*}, \bar{\psi}^{*}, \varphi^{*}, C^{*}\right\}$

are used. Independence of the functionals $\tilde{P}^{(k)}$ of the fields $\bar{C}^{\alpha}$ and the relations

$$
\begin{gathered}
P^{(k)} \int d x\left[\frac{\overleftarrow{\delta}}{\delta \mathcal{B}_{\mu}^{\beta}} D_{\mu}^{\beta \alpha}(\mathcal{B})+g \varepsilon^{\beta \gamma \alpha}\left(\frac{\overleftarrow{\delta}}{\delta \bar{C}^{\beta}} \bar{C}^{\gamma}+\frac{\overleftarrow{\delta}}{\delta A_{\mu}^{* \beta}} A_{\mu}^{* \gamma}\right)\right] \\
=\tilde{P}^{(k)} \int d x\left[\frac{\overleftarrow{\delta}}{\delta \mathcal{B}_{\mu}^{\beta}} D_{\mu}^{\beta \alpha}(\mathcal{B})+g \varepsilon^{\beta \gamma \alpha} \frac{\overleftarrow{\delta}}{\delta \mathcal{A}_{\mu}^{* \beta}} \mathcal{A}_{\mu}^{* \gamma}\right]
\end{gathered}
$$

allow us to write down the following set of equations for $\tilde{P}^{(k)}$

$$
\begin{aligned}
& \int d x\left[\tilde{P}^{(1)} \frac{\overleftarrow{\delta}}{\delta \Omega} \frac{\delta}{\delta \Omega^{*}} \tilde{P}^{(1)}-\theta_{\mu}^{\alpha} \frac{\delta}{\delta \mathcal{B}_{\mu}^{\alpha}} \tilde{P}^{(1)}\right]=0 \\
& 2 \xi \frac{\partial}{\partial \xi} \tilde{P}^{(1)}=\int d x\left[\tilde { P } ^ { ( 1 ) } \left(\frac{\overleftarrow{\delta}}{\delta \Omega} \frac{\delta}{\delta \Omega^{*}}\right.\right. \\
& \left.\left.-\frac{\overleftarrow{\delta}}{\delta \Omega^{*}} \frac{\delta}{\delta \Omega}\right) \tilde{P}^{(2)}-\theta_{\mu}^{\alpha} \frac{\delta}{\delta \mathcal{B}_{\mu}^{\alpha}} \tilde{P}^{(2)}\right] \\
& +\int d x\left[\left(\Omega^{*} \frac{\delta}{\delta \Omega^{*}}-\Omega \frac{\delta}{\delta \Omega}\right) \tilde{P}^{(1)}\right], \\
& \tilde{P}^{(k)} \overleftarrow{\tilde{h}^{\alpha}} \omega_{\alpha}=0, k=1,2
\end{aligned}
$$

where

$$
\begin{aligned}
& \overleftarrow{\tilde{h}^{\alpha}} \omega_{\alpha}=\int d x\left\{\left[\frac{\overleftarrow{\delta}}{\delta \mathcal{B}_{\mu}^{\beta}} D_{\mu}^{\beta \alpha}(\mathcal{B})+g \varepsilon^{\beta \gamma \alpha}\right.\right. \\
& \times\left(\frac{\overleftarrow{\delta}}{\delta \mathcal{A}_{\mu}^{\beta}} \mathcal{A}_{\mu}^{\gamma}+\frac{\overleftarrow{\delta}}{\delta \varphi^{\beta}} \varphi^{\gamma}+\frac{\overleftarrow{\delta}}{\delta C^{\beta}} C^{\gamma}\right) \\
& +g \varepsilon^{\beta \gamma \alpha}\left(\frac{\overleftarrow{\delta}}{\delta \mathcal{A}_{\mu}^{* \beta}} \mathcal{A}_{\mu}^{* \gamma}+\frac{\overleftarrow{\delta}}{\delta \varphi^{* \beta}} \varphi^{* \gamma}\right. \\
& \left.+\frac{\overleftarrow{\delta}}{\delta C^{* \beta}} C^{* \gamma}+\frac{\overleftarrow{\delta}}{\delta \theta_{\mu}^{\beta}} \theta_{\mu}^{\gamma}\right) \\
& -g t_{j k}^{\alpha}\left(\frac{\overleftarrow{\delta}}{\delta \psi_{j}} \psi_{k}+{\frac{\overleftarrow{\delta}}{\delta \bar{\psi}_{j}^{*}}}_{\psi_{k}^{*}}^{*}\right) \\
& \left.\left.+g\left(\frac{\overleftarrow{\delta}}{\delta \bar{\psi}_{j}} \bar{\psi}_{k}+\frac{\overleftarrow{\delta}}{\delta \psi_{j}^{*}} \psi_{k}^{*}\right) t_{k j}^{\alpha}\right] \omega_{\alpha}\right\}
\end{aligned}
$$

When studying the structure of functionals $\tilde{P}^{(k)}$ and in further research, it is helpful to have a consequence from equation (3.12) that corresponds to the case when $\omega_{\alpha}=$ const, 
$\tilde{P}^{(k)} \overleftarrow{T^{\alpha}}=0, k=1,2$

where

$$
\begin{aligned}
\overleftarrow{T^{\alpha}}= & \int d x\left\{\varepsilon ^ { \beta \gamma \alpha } \left(\frac{\overleftarrow{\delta}}{\delta \mathcal{B}_{\mu}^{\beta}} \mathcal{B}_{\mu}^{\gamma}\right.\right. \\
& \left.+\frac{\overleftarrow{\delta}}{\delta \mathcal{A}_{\mu}^{\beta}} \mathcal{A}_{\mu}^{\gamma}+\frac{\overleftarrow{\delta}}{\delta \varphi^{\beta}} \varphi^{\gamma}+\frac{\overleftarrow{\delta}}{\delta C^{\beta}} C^{\gamma}\right) \\
& +\varepsilon^{\beta \gamma \alpha}\left(\frac{\overleftarrow{\delta}}{\delta \mathcal{A}_{\mu}^{* \beta}} \mathcal{A}_{\mu}^{* \gamma}+\frac{\overleftarrow{\delta}}{\delta \varphi^{* \beta}} \varphi^{* \gamma}\right. \\
& \left.+\frac{\overleftarrow{\delta}}{\delta C^{* \beta}} C^{* \gamma}+\frac{\overleftarrow{\delta}}{\delta \theta_{\mu}^{\beta}} \theta_{\mu}^{\gamma}\right) \\
& -t_{j k}^{\alpha}\left(\frac{\overleftarrow{\delta}}{\delta \psi_{j}} \psi_{k}+\frac{\overleftarrow{\delta}_{\delta \bar{\psi}_{j}^{*}}^{*}}{\psi_{k}^{*}}\right) \\
& \left.+\left(\frac{\overleftarrow{\delta}}{\delta \bar{\psi}_{j}} \bar{\psi}_{k}+\frac{\overleftarrow{\delta}_{\delta \psi_{j}^{*}}^{*}}{\psi_{k}^{*}}\right) t_{k j}^{\alpha}\right\} .
\end{aligned}
$$

We refer to the equation of the form (3.14) as the ones of the $T$-symmetry for the corresponding functionals.

Using the properties of the functional $\tilde{P}^{(2)}$ : its locality and (3.4) as well as axial symmetry, Poincare- and $T$-symmetries, we find the general representation of $\tilde{P}^{(2)}$

$\tilde{P}^{(2)}=\sum_{k=1}^{5} Z_{k} \Gamma_{0 \mid k}+\int d x\left[Z_{1}^{\prime} \mathcal{A}_{\mu}^{* \alpha} \mathcal{B}_{\mu}^{\alpha}\right]$,

where $Z_{i}, i=1,2,3,4,5$ and $Z_{1}^{\prime}$ are arbitrary constants and the functionals $\Gamma_{0 \mid k}, k=1,2,3,4,5$ were introduced in (2.27), (2.28). Later on any quantities " $Z$ " with any set of indices do not depend on coordinates $x$ and field variables.

Further, when using the Eq. (3.12) for $\tilde{P}^{(2)}$, we get that $Z_{1}^{\prime}=0$. The final expression for the $\tilde{P}^{(2)}$ has the form

$\tilde{P}^{(2)}=\sum_{k=1}^{5} Z_{k} \Gamma_{0 \mid k}$,

Notice that the functional $\tilde{P}^{(2)}$ does not depend on the fields $\theta_{\mu}^{\alpha}$ and $\mathcal{B}_{\mu}^{\alpha}$. By taking (3.17) into account the equation (3.11) reduces to the following one

$$
\begin{aligned}
2 \xi \frac{\partial}{\partial \xi} \tilde{P}^{(1)}= & \hat{L}(Z) \tilde{P}^{(1)}, \\
\hat{L}(Z)= & \int d x\left[\left(Z_{1}-1\right)\left(\mathcal{A}_{\mu}^{\alpha} \frac{\delta}{\delta \mathcal{A}_{\mu}^{\alpha}}-\mathcal{A}_{\mu}^{* \alpha} \frac{\delta}{\delta \mathcal{A}_{\mu}^{* \alpha}}\right)\right. \\
& +\left(Z_{2}-1\right)\left(C^{\alpha} \frac{\delta}{\delta C^{\alpha}}-C^{* \alpha} \frac{\delta}{\delta C^{* \alpha}}\right) \\
& +\left(Z_{3}-1\right)\left(\psi_{j} \frac{\delta}{\delta \psi_{j}}-\psi_{j}^{*} \frac{\delta}{\delta \psi_{j}^{*}}\right) \\
& +\left(Z_{4}-1\right)\left(\bar{\psi}_{j} \frac{\delta}{\delta \bar{\psi}_{j}}-\bar{\psi}_{j}^{*} \frac{\delta}{\delta \bar{\psi}_{j}^{*}}\right)
\end{aligned}
$$

$$
\left.+\left(Z_{5}-1\right)\left(\varphi^{\alpha} \frac{\delta}{\delta \varphi^{\alpha}}-\varphi^{* \alpha} \frac{\delta}{\delta \varphi^{* \alpha}}\right)\right],
$$

describing the dependence of renormalization constants on the gauge parameter $\xi$. We refer to the Eq. (3.10) as the extended master-equation and to (3.18) as the gauge dependence equation.

\subsection{Solution to the extended master-equation}

Let us consider a solution to the extended master-equation (3.10) for the functional $\tilde{P}^{(1)}$ presented in the form

$$
\tilde{P}^{(1)}=\tilde{P}_{\theta}^{(1)}+\tilde{P}_{\Omega^{*}}^{(1)}+\tilde{P}_{\psi}^{(1)}+\tilde{P}_{\varphi}^{(1)}+\tilde{P}_{\psi \varphi}^{(1)}+\tilde{P}_{\mathcal{A B}}^{(1)} .
$$

Functional $\tilde{P}_{\theta}^{(1)}$ is written as

$\tilde{P}_{\theta}^{(1)}=\int d x \theta_{\mu}^{\alpha}(x) \tilde{P}_{\theta \mu}^{\alpha}(x)$,

and functionals $\tilde{P}_{\Omega^{*}}^{(1)}, \tilde{P}_{\psi}^{(1)}, \tilde{P}_{\varphi}^{(1)}, \tilde{P}_{\psi \varphi}^{(1)}, \tilde{P}_{\mathcal{A B}}^{(1)}$ do not depend on the fields $\theta_{\mu}^{\alpha}$. Taking into account the properties $\operatorname{dim}\left(\tilde{P}_{\theta \mu}^{\alpha}\right)=$ $2, \operatorname{gh}\left(\tilde{P}_{\mu \theta}^{\alpha}\right)=-1, \varepsilon\left(\tilde{P}_{\theta \mu}^{\alpha}\right)=1, \varepsilon_{f}\left(\tilde{P}_{\theta \mu}^{\alpha}\right)=0$ as well as the Poincare- and $T$-symmetries of $\tilde{P}_{\theta}^{(1)}$, we find that

$\tilde{P}_{\theta \mu}^{\alpha}(x)=-Z_{6} \mathcal{A}_{\mu}^{* \alpha}(x), \quad \tilde{P}_{\theta}^{(1)}=Z_{6} \Gamma_{0 \mid 6}$,

where $Z_{6}$ is an arbitrary constant.

Functional $\tilde{P}_{\Omega^{*}}^{(1)}(3.8)$ is linear in antifields $\Omega^{*}$ and functionals $\tilde{P}_{\psi}^{(1)}, \tilde{P}_{\varphi}^{(1)}, \tilde{P}_{\psi \varphi}^{(1)}, \tilde{P}_{\mathcal{A B}}^{(1)}$ do not depend on antifields $\Omega^{*}$. The functional $\tilde{P}_{\Omega^{*}}^{(1)}$ can be presented in the form

$\tilde{P}_{\Omega^{*}}^{(1)}=\tilde{P}_{\mathcal{A}^{*}}^{(1)}+\tilde{P}_{C^{*}}^{(1)}+\tilde{P}_{\psi^{*}}^{(1)}+\tilde{P}_{\bar{\psi}^{*}}^{(1)}+\tilde{P}_{\varphi^{*}}^{(1)}$.

Using the arguments similar to those that led us to establish the form of the functional $\tilde{P}_{\theta}^{(1)}(3.22)$, we obtain

$\tilde{P}_{\mathcal{A}^{*}}^{(1)}=Z_{7} \Gamma_{0 \mid 7}+Z_{8} \Gamma_{0 \mid 8}, \quad \tilde{P}_{C^{*}}^{(1)}=Z_{9} \Gamma_{0 \mid 9}$,

$\tilde{P}_{\psi^{*}}^{(1)}=Z_{10} \Gamma_{0 \mid 10}, \quad \tilde{P}_{\bar{\psi}^{*}}^{(1)}=Z_{11} \Gamma_{0 \mid 11}$,

$\tilde{P}_{\varphi^{*}}^{(1)}=Z_{12} \Gamma_{0 \mid 12}$.

Functional $\tilde{P}_{\psi}^{(1)}$ depends on the variables $\Psi, \mathcal{A}, \mathcal{B}$, and is quadratic in $\Psi$. Taking into account the axial symmetry as well as the Poincare- and $\mathcal{B}$-gauge invariance, we find the general structure of $\tilde{P}_{\psi}^{(1)}$,

$\tilde{P}_{\psi}^{(1)}=Z_{13} \Gamma_{0 \mid 13}+Z_{14} \Gamma_{0 \mid 14}+Z_{15} \Gamma_{0 \mid 15}$.

Functional $\tilde{P}_{\varphi}^{(1)}$ depends on the variables $\varphi, \mathcal{A}, \mathcal{B}$, and vanishes when $\varphi=0$. Taking into account the Poincare- and the $\mathcal{B}$-gauge invariance, we establish the general form of the functional $\tilde{P}_{\varphi}^{(1)}$,

$$
\begin{aligned}
\tilde{P}_{\varphi}^{(1)}= & Z_{16} \Gamma_{0 \mid 16}+Z_{17} \Gamma_{0 \mid 17} \\
& +\tilde{P}_{\varphi 18}^{(1)}+Z_{19} \Gamma_{0 \mid 19}+Z_{20} \Gamma_{0 \mid 20},
\end{aligned}
$$


where

$$
\begin{gathered}
\tilde{P}_{\varphi 18}^{(1)}=\int d x\left[\frac{g^{2}}{2} Z_{18}^{\alpha \beta \gamma \sigma} \mathcal{A}_{\mu}^{\alpha} \varphi^{\beta} \mathcal{A}_{\mu}^{\gamma} \varphi^{\sigma}\right], \\
Z_{18}^{\alpha \beta \gamma \sigma}=Z_{18}^{\gamma \beta \alpha \sigma}=Z_{18}^{\alpha \sigma \gamma \beta} .
\end{gathered}
$$

If the condition

$$
\begin{aligned}
& \int d x\left[Z_{18}^{\alpha \beta \gamma \sigma} \mathcal{A}_{\mu}^{\alpha} \varphi^{\beta} \mathcal{A}_{\mu}^{\gamma} \varphi^{\sigma}\right] \\
& =Z_{18} \int d x\left[\sum_{\sigma=1}^{3} E_{\sigma \beta}^{\alpha} E_{\sigma \rho}^{\gamma} \mathcal{A}_{\mu}^{\alpha} \varphi^{\beta} \mathcal{A}_{\mu}^{\gamma} \varphi^{\rho}\right]
\end{aligned}
$$

fulfils then the functional $\tilde{P}_{\varphi 18}^{(1)}$ satisfies the Eq. (3.12).

Functional $\tilde{P}_{\psi \varphi}^{(1)}$ is an interaction vertex of the fields $\varphi$ and $\Psi$. Taking into account the axial, Poincare- and $T$ symmetries, this functional has the following general form:

$\tilde{P}_{\psi \varphi}^{(1)}=Z_{21} \Gamma_{0 \mid 21}$

The functional $\tilde{P}_{\psi \varphi}^{(1)}(3.30)$ satisfies the Eq. (3.12).

Substitute into the Eq. (3.10) the representation for the functional $\tilde{P}^{(1)}$ in the form (3.20) where the terms $\tilde{P}_{\theta}^{(1)}, \tilde{P}_{\Omega^{*}}^{(1)}$, $\tilde{P}_{\psi}^{(1)}, \tilde{P}_{\varphi}^{(1)}$ and $\tilde{P}_{\psi \varphi}^{(1)}$ are described by the relations (3.22), (3.23)-(3.25), (3.26), (3.27)-(2.33) and (3.30), respectively, and take into account that $\tilde{P}_{\mathcal{A B}}^{(1)}=\tilde{P}_{\mathcal{A B}}^{(1)}(\mathcal{A}, \mathcal{B})$. Then, a solution to the Eq. (3.10) is reduced to solutions to the subequations which follow from the requirement for independent polynomial structures appearing in the left-hand side of the Eq. (3.10), to be equal to zero. In their turn, these subequations are reduced to algebraic equations for coefficients " $Z$ " or, in two cases, to variational differential equations for the functional $\tilde{P}_{\mathcal{A B}}^{(1)}(\mathcal{A}, \mathcal{B})$.

We explain the results obtained by using an example for the block

$$
\begin{aligned}
& \theta \mathcal{A}^{*} C \Rightarrow \int d x\left[Z_{6} Z_{8} \Gamma_{0 \mid 8} \frac{\overleftarrow{\delta}}{\delta \mathcal{A}_{\mu}^{\alpha}}\right. \\
& \left.\frac{\delta}{\delta \mathcal{A}_{\mu}^{* \alpha}} \Gamma_{0 \mid 6}-Z_{7} \theta_{\mu}^{\alpha} \frac{\delta}{\delta \mathcal{B}_{\mu}^{\alpha}} \Gamma_{0 \mid 7}\right]=0 \Rightarrow \\
& Z_{8}=\frac{Z_{7}}{Z_{6}}
\end{aligned}
$$

This block should be understood as the following: the requirement for the structure $\theta \mathcal{A}^{*} C$ to be equal to zero leads to equation (3.31), from which it follows the relation (3.32).
Further

$$
\begin{gathered}
\theta \bar{\psi} \gamma^{\mu} \psi \Rightarrow \int d x\left[Z_{6} Z_{14} \Gamma_{0 \mid 14} \frac{\overleftarrow{\delta}}{\delta \mathcal{A}_{\mu}^{\alpha}} \frac{\delta}{\delta \mathcal{A}_{\mu}^{* \alpha}}\right. \\
\left.\Gamma_{0 \mid 6}-Z_{13} \theta_{\mu}^{\alpha} \frac{\delta}{\delta \mathcal{B}_{\mu}^{\alpha}} \Gamma_{0 \mid 13}\right]=0 \Rightarrow \\
Z_{14}=\frac{Z_{13}}{Z_{6}} ; \\
\theta \varphi(D(\mathcal{B}) \varphi) \Rightarrow \int d x\left[Z_{6} Z_{17} \Gamma_{0 \mid 17} \frac{\overleftarrow{\delta}}{\delta \mathcal{A}_{\mu}^{\alpha}} \frac{\delta}{\delta \mathcal{A}_{\mu}^{* \alpha}}\right. \\
\left.\Gamma_{0 \mid 6}-Z_{16} \theta_{\mu}^{\alpha} \frac{\delta}{\delta \mathcal{B}_{\mu}^{\alpha}} \Gamma_{0 \mid 16}\right]=0 \Rightarrow \\
Z_{17}=\frac{Z_{16}}{Z_{6}} ; \\
\theta \mathcal{A} \varphi \varphi \Rightarrow \int d x\left[Z_{6} \tilde{P}_{\varphi 18}^{(1)} \frac{\overleftarrow{\delta}}{\delta \mathcal{A}_{\mu}^{\alpha}} \frac{\delta}{\delta \mathcal{A}_{\mu}^{* \alpha}}\right. \\
\left.\Gamma_{0 \mid 6}-Z_{17} \theta_{\mu}^{\alpha} \frac{\delta}{\delta \mathcal{B}_{\mu}^{\alpha}} \Gamma_{0 \mid 17}\right]=0 \Rightarrow \\
Z_{18}^{\alpha \beta \gamma \sigma} \varphi^{\beta} \mathcal{A}_{\mu}^{\gamma} \varphi^{\sigma}=\frac{Z_{17}}{Z_{6}} E_{\alpha \beta}^{\rho} E_{\gamma \sigma}^{\rho} \varphi^{\beta} \mathcal{A}_{\mu}^{\gamma} \varphi^{\sigma}
\end{gathered}
$$

Multiplying the equality (3.35) by $\mathcal{A}_{\mu}^{\alpha}$ and integrating then over $x$, we find that the relation (3.29) is satisfied and the functional $\tilde{P}_{\varphi 18}^{(1)}$ reads

$$
\begin{gathered}
\tilde{P}_{\varphi 18}^{(1)}=Z_{18} \Gamma_{0 \mid 18}, \quad Z_{18}=\frac{Z_{17}}{Z_{6}}=\frac{Z_{16}}{Z_{6}^{2}} \\
\theta \mathcal{A}^{n} \mathcal{B}^{k} \Rightarrow \int d x\left[Z_{6} \tilde{P}_{\mathcal{A} \mathcal{B}}^{(1)} \frac{\overleftarrow{\delta}}{\delta \mathcal{A}_{\mu}^{\alpha}} \frac{\delta}{\delta \mathcal{A}_{\mu}^{* \alpha}}\right. \\
\left.\Gamma_{0 \mid 6}-\theta_{\mu}^{\alpha} \frac{\delta}{\delta \mathcal{B}_{\mu}^{\alpha}} \tilde{P}_{\mathcal{A} \mathcal{B}}^{(1)}\right]=0 \Rightarrow \\
\left(Z_{6} \frac{\delta}{\delta \mathcal{A}_{\mu}^{\alpha}}-\frac{\delta}{\delta \mathcal{B}_{\mu}^{\alpha}}\right) \tilde{P}_{\mathcal{A B}}^{(1)}=0 \Rightarrow \\
\tilde{P}_{\mathcal{A B}}^{(1)}=\tilde{P}_{\mathcal{A B}}^{(1)}(U), U=U\left(Z_{6}\right)=\mathcal{B}+\frac{1}{Z_{6}} \mathcal{A}
\end{gathered}
$$

$$
\begin{gathered}
\mathcal{A}^{*} \mathcal{A} C C \Rightarrow \int d x\left[Z_{8}^{2} \Gamma_{0 \mid 8} \frac{\overleftarrow{\delta}}{\delta \mathcal{A}_{\mu}^{\alpha}} \frac{\delta}{\delta \mathcal{A}_{\mu}^{* \alpha}}\right. \\
\left.\Gamma_{0 \mid 8}+Z_{8} Z_{9} \Gamma_{0 \mid 8} \frac{\overleftarrow{\delta}}{\delta C^{\alpha}} \frac{\delta}{\delta C^{* \alpha}} \Gamma_{0 \mid 9}\right]=0 \Rightarrow \\
Z_{9}=Z_{8}=\frac{Z_{7}}{Z_{6}}
\end{gathered}
$$$$
\psi^{*} \psi C C \Rightarrow \int d x\left[Z_{10}^{2} \Gamma_{0 \mid 10} \frac{\overleftarrow{\delta}}{\delta \psi_{j}} \frac{\delta}{\delta \psi_{j}^{*}}\right.
$$$$
\left.\Gamma_{0 \mid 10}+Z_{10} Z_{9} \Gamma_{0 \mid 10} \frac{\overleftarrow{\delta}}{\delta C^{\alpha}} \frac{\delta}{\delta C^{* \alpha}} \Gamma_{0 \mid 9}\right]=0 \Rightarrow
$$$$
Z_{10}=Z_{9}=\frac{Z_{7}}{Z_{6}}
$$ 


$$
\begin{aligned}
& \overline{\psi \psi}^{*} C C \Rightarrow \int d x\left[Z_{11}^{2} \Gamma_{0 \mid 11} \frac{\overleftarrow{\delta}}{\delta \bar{\psi}_{j}} \frac{\delta}{\delta \bar{\psi}_{j}^{*}}\right. \\
& \left.\Gamma_{0 \mid 11}+Z_{11} Z_{9} \Gamma_{0 \mid 11} \frac{\overleftarrow{\delta}}{\delta C^{\alpha}} \frac{\delta}{\delta C^{* \alpha}} \Gamma_{0 \mid 9}\right]=0 \Rightarrow \\
& Z_{11}=Z_{9}=\frac{Z_{7}}{Z_{6}} \\
& \bar{\psi} \psi \varphi C \Rightarrow \int d x\left[Z_{10} Z_{21} \Gamma_{0 \mid 21} \frac{\overleftarrow{\delta}}{\delta \psi_{j}} \frac{\delta}{\delta \psi_{j}^{*}}\right. \\
& \Gamma_{0 \mid 10}+Z_{11} Z_{21} \Gamma_{0 \mid 21} \frac{\overleftarrow{\delta}}{\delta \bar{\psi}_{j}} \frac{\delta}{\delta \bar{\psi}_{j}^{*}} \Gamma_{0 \mid 11} \\
& \left.+Z_{12} Z_{21} \Gamma_{0 \mid 21} \frac{\overleftarrow{\delta}}{\delta \varphi^{\alpha}} \frac{\delta}{\delta \varphi^{* \alpha}} \Gamma_{0 \mid 12}\right]=0 \Rightarrow \\
& Z_{12}=Z_{10}=\frac{Z_{7}}{Z_{6}} \\
& \mathcal{A}^{n} \mathcal{B}^{k} C \Rightarrow \int d x\left[\tilde{P}_{\mathcal{A} \mathcal{B}}^{(1)} \frac{\overleftarrow{\delta}}{\delta \mathcal{A}_{\mu}^{\alpha}} \frac{\delta}{\delta \mathcal{A}_{\mu}^{* \alpha}}\right. \\
& \left.\left(Z_{7} \Gamma_{0 \mid 7}+Z_{8} \Gamma_{0 \mid 8}\right)\right]=0 \\
& \Rightarrow D_{\mu}^{\alpha \beta}(U) \frac{\delta}{\delta U_{\mu}^{\beta}} \tilde{P}_{\mathcal{A B}}^{(1)}(U)=0 \Rightarrow \tilde{P}_{\mathcal{A B}}^{(1)}=Z_{22} \Gamma_{0 \mid 22}\left(Z_{6}\right),
\end{aligned}
$$$$
\Gamma_{0 \mid 22}\left(Z_{6}\right)=-\frac{1}{4} \int d x\left[G_{\mu \nu}^{\alpha}\left(U\left(Z_{6}\right)\right) G_{\mu \nu}^{\alpha}\left(U\left(Z_{6}\right)\right)\right] .
$$

Requirement for the rest structures in the left-hand side of the Eq. (3.10) to be equal to zero is satisfied identically.

Notice that the functional $\tilde{P}^{(1)}$ can be represented as a linear combination of independent polynomials $\Gamma_{0 \mid k}$,

$\tilde{P}^{(1)}=\sum_{k=6}^{22} Z_{k} \Gamma_{0 \mid k}$

\subsection{Solution to the gauge dependence equation}

Consider now a solution to the Eq. (3.18) describing the dependence of constants " $Z$ " on the gauge parameter $\xi$ appearing in the general solution to the extended masterequation (3.10) for the functional $\tilde{P}^{(1)}$. For the functional $\tilde{P}^{(1)}$ we will use the representation (3.44). Notice that every functional $\Gamma_{0 \mid k}, k=6, \ldots, 21$ is eigen for the operator $\hat{L}$ (3.19),

$\hat{L} \Gamma_{0 \mid k}=\zeta_{k} \Gamma_{0 \mid k}$,

where $\zeta_{k}$ are eigenvalues of the operator $\hat{L}$. It means that the Eq. (3.18) reduces to the set of equations of the form

$2 \xi \dot{Z}_{k}=\zeta_{k}, k=6, \ldots, 21$.
In relations (3.46) and later on for any quantity $I=I(\xi, \ldots)$ we use the notation

$\dot{I} \equiv \frac{\partial}{\partial \xi} I$.

Equation (3.46) for independent constants $Z_{k}$ lead to the following relations and consequences,

$$
\begin{aligned}
k= & 6, \quad 2 \xi \dot{Z}_{6}=Z_{6}\left(1-Z_{1}\right) \\
& \Rightarrow \quad Z_{1}=1-2 \xi \frac{\dot{Z}_{6}}{Z_{6}}, \\
k= & 7, \quad 2 \xi \dot{Z}_{7}=Z_{7}\left(Z_{2}-Z_{1}\right) \\
& \Rightarrow \quad Z_{2}=1+2 \xi\left(\frac{\dot{Z}_{7}}{Z_{7}}-\frac{\dot{Z}_{6}}{Z_{6}}\right), \\
k= & 13, \quad 2 \xi \dot{Z}_{13}=Z_{13}\left(Z_{3}+Z_{4}-2\right) \\
& \Rightarrow \quad Z_{4}=2+2 \xi \frac{\dot{Z}_{13}}{Z_{13}}-Z_{3}, \\
k= & 15, \quad 2 \xi \dot{Z}_{15}=Z_{15}\left(Z_{3}+Z_{4}-2\right) \\
& \Rightarrow \quad \dot{Z}_{23}=0, \quad Z_{15}=Z_{13} Z_{23}, \\
k= & 16, \quad \xi \dot{Z}_{16}=Z_{16}\left(Z_{5}-1\right) \\
& \Rightarrow \quad Z_{5}=1+\xi \frac{Z_{16}}{Z_{16}}, \\
k= & 19, \quad \xi \dot{Z}_{19}=Z_{19}\left(Z_{5}-1\right) \\
& \Rightarrow \dot{Z}_{24}=0, \quad Z_{19}=Z_{16} Z_{24}, \\
k= & 20, \quad \xi \dot{Z}_{20}=2 Z_{20}\left(Z_{5}-1\right) \\
& \Rightarrow \dot{Z}_{25}=0, \quad Z_{20}=Z_{16}^{2} Z_{25}, \\
k= & 21, \quad 2 \xi \dot{Z}_{21}=Z_{21}\left(Z_{3}+Z_{4}+Z_{5}-3\right) \\
& \Rightarrow \dot{Z}_{26}=0, Z_{21}=Z_{13} Z_{16}^{1 / 2} Z_{26} .
\end{aligned}
$$

As to the functional $\Gamma_{0 \mid 22}\left(Z_{6}\right)$, let us represent it in the form

$\Gamma_{0 \mid 22}\left(Z_{6}\right)=\sum_{l=0}^{4} Z_{6}^{-l} \Gamma_{0 \mid 22, l}$.

Then, from the Eq. (3.18), it follows

$$
\begin{aligned}
& l=0, \quad \dot{Z}_{22}=0, \\
& l=1,2,3,4, \quad-2 \xi \frac{\dot{Z}_{6}}{Z_{6}}=Z_{1}-1 .
\end{aligned}
$$

The last relation is equivalent to (3.48).

Below, in Sect. 5, we find that all constants " $Z$ " can be interpreted as renormalization constants which are uniquely defined from the conditions of reducing divergences.

Now we formulate the results obtained in this section as the following lemma.

Lemma: Let

$P=\int d x P\left(Q, Q^{*}, \bar{C}, B, \mathcal{B}, \xi, \theta, \chi\right)$,

be a local functional of all variables with quantum numbers of the action $S_{\text {ext }}$ and satisfies all Eqs. (2.19)-(2.24) and 
additional symmetries (the axial- , Poincare - invariance and so on) which were used in solving the Eqs. (2.19)-(2.24) (with substitution $S_{\text {ext }} \rightarrow P$ ). Then the functional $P$ has the form

$$
\begin{aligned}
P= & \Gamma_{0 \mid 0}+\sum_{k=6}^{22} Z_{k} \Gamma_{0 \mid k}+\chi \sum_{k=1}^{5} Z_{k} \Gamma_{0 \mid k}, \\
Z_{8}= & Z_{9}=Z_{10}=Z_{11}=Z_{12}=\frac{Z_{7}}{Z_{6}}, \\
Z_{14}= & \frac{Z_{13}}{Z_{6}}, Z_{15}=Z_{13} Z_{23}, Z_{17}=\frac{Z_{16}}{Z_{6}}, \\
Z_{18}= & \frac{Z_{16}}{Z_{6}^{2}}, Z_{19}=Z_{16} Z_{24}, Z_{20}=Z_{16}^{2} Z_{25}, \\
Z_{21}= & Z_{13} Z_{16}^{1 / 2} Z_{26}, \\
Z_{1}= & 2 \xi \frac{Z_{6}}{Z_{6}}, Z_{2}=1+2 \xi\left(\frac{\dot{Z}_{7}}{Z_{7}}-\frac{\dot{Z}_{6}}{Z_{6}}\right), \\
Z_{4}= & 2+2 \xi \frac{\dot{Z}_{13}}{Z_{13}}-Z_{3}, \\
Z_{5}= & 1+2 \xi \frac{\dot{Z}_{16}}{Z_{16}}, \quad \dot{Z}_{22}=\dot{Z}_{23}=\dot{Z}_{24}=\dot{Z}_{25}=\dot{Z}_{26}=0,
\end{aligned}
$$

where $Z_{6}, Z_{7}, Z_{13}, Z_{15}, Z_{16}, Z_{19}, Z_{20}, Z_{21}, Z_{22}$ are arbitrary constants depending perhaps on $\xi$, the constants $Z_{22}, Z_{23}, Z_{24}, Z_{25}, Z_{26}$ do not depend on $\xi$. Among the set of indices of all constants " $Z$ ", $\{k\}=1, \ldots, 26$ we will highlight the three groups: the first is indices of independent constants,

$\left\{k_{\text {ind }}\right\}=3,6,7,13,16,22,23,24,25,26$;

the second is indices of constants independent of $\xi$,

$\left\{k_{c o n}\right\}=22,23,24,25,26 \subset\left\{k_{\text {ind }}\right\}$;

and the third is indices of dependent constants,

$\left\{k_{d e p}\right\}=1,2,4,5,8,9,10,11,12,14,15,17,18,19,20,21$.

It should be noted that the functional $P$ does not contain vertices additional to that from which the action $S_{\text {ext }}(2.9)$ is built up. Namely, the obvious relation is valid,

$S_{\text {ext }}=\left.P\right|^{\prime \prime} Z^{\prime \prime}=1$
In its turn, the functional $P(3.60)$ can be represented in the form analogous to (2.9) for the action $S_{\text {ext }}$,

$$
\begin{aligned}
P= & \int d x\left\{-\frac{Z_{22}}{4} G_{\mu \nu}^{\alpha}(U) G_{\mu \nu}^{\alpha}(U)+Z_{13} i \bar{\psi} \gamma^{\mu}\right. \\
& \times D_{\psi \mu}(U) \psi+\frac{Z_{16}}{2}\left(D_{\varphi \mu}^{\alpha \beta}(U) \varphi^{\beta}\right) D_{\varphi \mu}^{\alpha \gamma}(U) \varphi^{\gamma} \\
& -Z_{15} m \bar{\psi} \psi-Z_{19} \frac{M^{2}}{2} \varphi^{2}-Z_{20} \frac{\lambda}{4} \varphi^{4} \\
& +Z_{21} i \vartheta \varphi^{\alpha} \bar{\psi} t^{\alpha} \psi+Z_{6} \theta_{\mu}^{\alpha}\left[D_{\mu}^{\alpha \beta}(U) \bar{C}^{\beta}-A_{\mu}^{* \alpha}\right] \\
& +\frac{Z_{7}}{Z_{6}} Q^{*} \mathcal{T}_{Q}+\chi\left[Z_{1}\left(A_{\mu}^{* \alpha}-D_{\mu}^{\alpha \beta}(\mathcal{B}) \bar{C}^{\beta}\right) A_{\mu}^{\alpha}\right. \\
& \left.+Z_{2} C^{* \alpha} C^{\alpha}+Z_{3} \psi^{*} \psi+Z_{4} \bar{\psi}^{*} \bar{\psi}+Z_{5} \varphi^{* \alpha} \varphi^{\alpha}\right] \\
& +(\xi / 2) B^{\alpha} B^{\alpha}+B^{\alpha} D_{\mu}^{\alpha \beta}(\mathcal{B}) A_{\mu}^{\beta} \\
& \left.+Z_{7} \bar{C}^{\alpha} D_{\mu}^{\alpha \beta}(\mathcal{B}) D_{\mu}^{\beta \gamma}(U) C^{\gamma}\right\}, \\
\mathcal{T}_{A_{\mu}^{\alpha}}= & Z_{6} D_{\mu}^{\alpha \beta}(U) C^{\beta}, \quad \mathcal{T}_{\psi, \bar{\psi}, \varphi, C}=\mathcal{R}_{\psi, \bar{\psi}, \varphi, C} .
\end{aligned}
$$

Representation (3.67) for the functional $P$ shows that it can be considered as (renormalized) action of the Yang-Mills theory, and from representations (3.60) and (3.66) of functionals $P$ and $S_{\text {ext }}$ it follows that the renormalization is multiplicative.

The inverse (maybe trivial but important) statement is valid: if the functional $P$ has the form (3.60) and the relations (3.61), (3.62) are satisfied then this functional satisfies the Eqs. (2.20)-(2.24).

\section{Effective action}

It is useful to define the generating functional of Green functions with the help of the functional $P$ constructed in the previous section, because it allows one to obtain a finite theory from the beginning. In what follows we redefine the functional $P, P \equiv S_{R}$, and, respectively, $P^{(k)} \equiv S_{R}^{(k)}$, $\tilde{P}^{(k)} \equiv \tilde{S}_{R}^{(k)}, k=1,2$.

The generating functional of Green functions is given by the following functional integral

$$
\begin{aligned}
Z\left(J_{\Phi}, L\right)= & \int d \Phi \exp \left(\frac{i}{\eta}\left[S_{R}+J_{\Phi} \Phi\right]\right) \\
& =\exp \left\{\frac{i}{\eta} W\left(J_{\Phi}, L\right)\right\},
\end{aligned}
$$

where $\eta$ is the parameter of a loop expansion of expression in the exponent (4.1), $W\left(J_{\Phi}, L\right)$ is the generating functional of connected Green functions, $J_{\Phi}$ is the set of sources to fields $\Phi$ and the notations $\Phi=\{Q, \bar{C}, B\}$ and $L=\left\{L^{A}\right\}=$ $\left\{\mathcal{B}, Q^{*}, \xi, \theta, \chi\right\}$ are introduced. We suppose also that all " $Z$ " are functions of $\eta,{ }^{\prime \prime} Z^{\prime \prime}={ }^{\prime \prime} Z(\eta)^{\prime \prime}$ expanding in the Taylor series, $Z_{k}(0)=1, \dot{Z}_{k}=O(\eta), k=1, \ldots, 26$. In that case the functional $S_{R}$ becomes a function of $\eta$, 
$S_{R}=S_{R}(\eta)=\sum_{l=0}^{\infty} \eta^{l} S_{R, l}, \quad S_{R}^{[k]}=\sum_{l=0}^{k} \eta^{l} S_{R, l}$,

and all functionals $S_{R, l}$ are linear combinations of the same set of polynomials $\left\{\Gamma_{0 \mid k}, k=0,1, \ldots, 22\right\}$ with the help of which the functionals $S_{\text {ext }}$ and $P$ are presented.

The generating functional of vertex functions (effective action) is defined with the help of the Legendre transformation,

$\Gamma\left(\Phi_{m \mid}, L\right)=W\left(J_{\Phi}, L\right)-J_{\Phi} \Phi_{m \mid}, \quad \Phi_{m \mid}=\frac{\delta}{\delta J_{\Phi}} W\left(J_{\Phi}, L\right)$,

with quantum numbers $\varepsilon(\Gamma)=0, \operatorname{gh}(\Gamma)=0, \operatorname{dim}(\Gamma)=0$, $\varepsilon_{f}(\Gamma)=0$ and satisfies the relations

$\Gamma\left(\Phi_{m \mid}, L\right) \frac{\overleftarrow{\delta}}{\delta \Phi_{m}}=-J_{\Phi}\left(\Phi_{m \mid}, L\right)$

$\Gamma\left(\Phi_{m \mid}, L\right) \frac{\overleftarrow{\delta}}{\delta L^{A}}=W\left(J_{\Phi}, L\right) \frac{\overleftarrow{\delta}}{\delta L^{A}}$

Functional averaging of Eqs. (2.20)-(2.24) with substitution $S_{\text {ext }} \rightarrow S_{R}$ leads to the equations for the functional $\Gamma=$ $\Gamma\left(\Phi_{m}, L\right)$ copying the equations for $S_{R}$,

$$
\begin{aligned}
& \int d x\left(\Gamma \frac{\overleftarrow{\delta}}{\delta Q_{m \mid}} \frac{\delta}{\delta Q^{*}} \Gamma-B_{m \mid} \frac{\delta}{\delta \bar{C}_{m \mid}} \Gamma-\theta \frac{\delta}{\delta \mathcal{B}} \Gamma\right) \\
& +2 \chi \xi \frac{\partial}{\partial \xi} \Gamma \\
& +\chi \int d x\left[\left(Q_{m \mid} \frac{\delta}{\delta Q_{m \mid}}-Q^{*} \frac{\delta}{\delta Q^{*}}\right.\right. \\
& \left.\left.-\bar{C}_{m \mid} \frac{\delta}{\delta \bar{C}_{m \mid}}-B_{m \mid} \frac{\delta}{\delta B_{m \mid}}\right) \Gamma\right]=0 \\
& \Gamma \overleftarrow{H_{m \mid}^{\alpha}} \omega_{\alpha}=0
\end{aligned}
$$

where $\overleftarrow{H_{m}^{\alpha}} \omega_{\alpha}$ is given by expression (2.21) with substitution $\Phi \rightarrow \Phi_{m \mid}$,

$$
\begin{aligned}
& \frac{\delta}{\delta B_{m \mid}^{\alpha}} \Gamma=D_{\mu}^{\alpha \beta}(\mathcal{B}) A_{m \mid \mu}^{\beta}+\xi B_{m \mid}^{\alpha}, \\
& D_{\mu}^{\alpha \beta}(\mathcal{B}) \frac{\delta}{\delta A_{\mu}^{* \beta}} \Gamma-\frac{\delta}{\delta \bar{C}_{m \mid}^{\alpha}} \Gamma=-g \varepsilon^{\alpha \beta \gamma} A_{m \mid \mu}^{\beta} \theta_{\mu}^{\gamma} .
\end{aligned}
$$

Represent the functional $\Gamma$ in the form

$\Gamma=\Gamma_{0 \mid 0}+\Gamma^{(1)}+\chi \Gamma^{(2)}$,

where the functionals $\Gamma^{(1)}$ and $\Gamma^{(2)}$ do not depend on the parameter $\chi$. Thanks to the structure chosen for the functional (4.9), from the Eqs. (4.7) and (4.8), it follows that the functionals $\Gamma^{(1)}$ and $\Gamma^{(2)}$ do not depend on the fields $B_{m \mid}^{\alpha}$,

$$
\begin{aligned}
& \frac{\delta}{\delta B_{m \mid}} \Gamma^{(k)}=0, \Gamma^{(k)} \\
& \quad=\Gamma^{(k)}\left(Q_{m \mid}, \bar{C}_{m \mid}, \mathcal{B}, Q^{*}, \xi, \theta\right), k=1,2,
\end{aligned}
$$

and satisfy the equations

$$
\left(D_{\mu}^{\alpha \beta}(\mathcal{B}) \frac{\delta}{\delta A_{\mu}^{* \beta}}-\frac{\delta}{\delta \bar{C}_{m \mid}^{\alpha}}\right) \Gamma^{(k)}=0, k=1,2 .
$$

In its turn, the Eq. (4.5) splits into two ones, one of them is closed with respect to $\Gamma^{(1)}$,

$$
\begin{gathered}
\int d x\left[\Gamma^{(1)} \frac{\overleftarrow{\delta}}{\delta Q_{m \mid}} \frac{\delta}{\delta Q^{*}} \Gamma^{(1)}-g \theta_{\mu}^{\alpha} \varepsilon^{\alpha \beta \gamma} \bar{C}_{m \mid}^{\beta}\right. \\
\left.\frac{\delta}{\delta A_{\mu}^{* \gamma}} \Gamma^{(1)}-\theta_{\mu}^{\alpha} \frac{\delta}{\delta \mathcal{B}_{\mu}^{\alpha}} \Gamma^{(1)}\right]=0
\end{gathered}
$$

and the second includes both functionals and describes their dependence on the gauge parameter $\xi$,

$$
\begin{aligned}
2 \xi \frac{\partial}{\partial \xi} \Gamma^{(1)}= & \int d x\left[\Gamma^{(1)}\left(\frac{\overleftarrow{\delta}}{\delta Q_{m \mid}} \frac{\delta}{\delta Q^{*}}-\frac{\overleftarrow{\delta}}{\delta Q^{*}} \frac{\delta}{\delta Q_{m \mid}}\right) \Gamma^{(2)}\right. \\
& \left.-\left(g \theta_{\mu}^{\alpha} \varepsilon^{\alpha \beta \gamma} \bar{C}_{m \mid}^{\beta} \frac{\delta}{\delta A_{\mu}^{* \gamma}}+\theta_{\mu}^{\alpha} \frac{\delta}{\delta \mathcal{B}_{\mu}^{\alpha}}\right) \Gamma^{(2)}\right] \\
& +\int d x\left[\left(\bar{C}_{m \mid} \frac{\delta}{\delta \bar{C}_{m \mid}}-Q_{m \mid} \frac{\delta}{\delta Q_{m} \mid}+Q^{*} \frac{\delta}{\delta Q^{*}}\right) \Gamma^{(1)}\right]
\end{aligned}
$$

Now, the Eq. (4.6) rewrites in the form of the two equations for the functionals $\Gamma^{(1)}$ and $\Gamma^{(2)}$,

$\Gamma^{(k)} \overleftarrow{h_{m \mid}^{\alpha}} \omega_{\alpha}=0, k=1,2$

where

$$
\begin{aligned}
\overleftarrow{h_{m \mid}^{\alpha}} \omega_{\alpha}= & \int d x\left\{\left[\frac{\overleftarrow{\delta}}{\delta \mathcal{B}_{\mu}^{\beta}} D_{\mu}^{\beta \alpha}(\mathcal{B})\right.\right. \\
& +g \varepsilon^{\beta \gamma \alpha}\left(\frac{\overleftarrow{\delta}}{\delta A_{m \mid \mu}^{\beta}} A_{m \mid \mu}^{\gamma}+\frac{\overleftarrow{\delta}}{\delta \varphi_{m \mid}^{\beta}} \varphi_{m \mid}^{\gamma}+\frac{\overleftarrow{\delta}}{\delta C_{m \mid}^{\beta}} C_{m \mid}^{\gamma}\right) \\
& +g \varepsilon^{\beta \gamma \alpha}\left(\frac{\overleftarrow{\delta}}{\delta \bar{C}_{m \mid}^{\beta}} \bar{C}_{m \mid}^{\gamma}+\frac{\overleftarrow{\delta}}{\delta A_{\mu}^{* \beta}} A_{\mu}^{* \gamma}+\frac{\overleftarrow{\delta}}{\delta C^{* \beta}} C^{* \gamma}\right. \\
& \left.+\frac{\overleftarrow{\delta}}{\delta \varphi^{* \beta}} \varphi^{* \gamma}+\frac{\overleftarrow{\delta}}{\delta \theta_{\mu}^{\beta}} \theta_{\mu}^{\gamma}\right) \\
& -g t_{j k}^{\alpha}\left(\frac{\overleftarrow{\delta}}{\delta \psi_{m \mid j}} \psi_{m \mid k}+\frac{\overleftarrow{\delta}}{\delta \bar{\psi}_{j}^{*}} \bar{\psi}_{k}^{*}\right)+g \\
& \left.\left.\left(\frac{\overleftarrow{\delta}}{\delta \bar{\psi}_{m \mid j}} \bar{\psi}_{m \mid k}+\frac{\overleftarrow{\delta}}{\delta \psi_{j}^{*}} \psi_{k}^{*}\right) t_{k j}^{\alpha}\right] \omega_{\alpha}\right\}
\end{aligned}
$$


Due to the Eq. (4.11) it is useful to introduce new variables $\mathcal{A}_{\mu}^{* \alpha}=\mathcal{A}_{\mu}^{* \alpha}(x), \mathcal{A}_{m \mid \mu}^{* \alpha}=\mathcal{A}_{m \mid \mu}^{* \alpha}(x)$,

$$
\begin{aligned}
\mathcal{A}_{\mu}^{* \alpha} & =A_{\mu}^{* \alpha}-D_{\mu}^{\alpha \beta}(\mathcal{B}) \bar{C}^{\beta} \\
\mathcal{A}_{m \mid \mu}^{* \alpha} & =A_{\mu}^{* \alpha}-D_{\mu}^{\alpha \beta}(\mathcal{B}) \bar{C}_{m \mid}^{\beta},
\end{aligned}
$$

and to use the following agreement for the sake of uniformity of further notations

$\mathcal{A}_{\mu}^{\alpha}=A_{\mu}^{\alpha}$

Also, let us introduce new functionals $\tilde{\Gamma}^{(k)}$ by the rule $\tilde{\Gamma}^{(k)}\left(\mathcal{B}, \bar{C}_{m \mid}, \mathcal{A}_{m \mid}^{*}, \Lambda_{m \mid}\right)=\Gamma^{(k)}\left(\mathcal{B}, \bar{C}_{m \mid}, A^{*}, \Lambda_{m \mid}\right)$

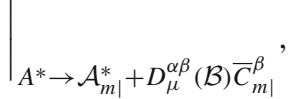

where the following notations

$\Lambda=\left\{Q, \psi^{*}, \bar{\psi}^{*}, C^{*}, \xi, \theta\right\}, \quad \Lambda_{m \mid}=\left\{Q_{m \mid}, \psi^{*}, \bar{\psi}^{*}, C^{*}, \xi, \theta\right\}$

were used. Taking into account the definitions (4.16)-(4.18) we have

$$
\begin{aligned}
& \frac{\delta}{\delta A_{\mu}^{* \alpha}} \Gamma^{(k)}=\frac{\delta}{\delta \mathcal{A}_{m \mid \mu}^{* \alpha}} \tilde{\Gamma}^{(k)}, \\
& \frac{\delta}{\delta \bar{C}_{m \mid}^{\alpha}} \Gamma^{(k)}=\frac{\delta}{\delta \bar{C}_{m \mid}^{\alpha}} \tilde{\Gamma}^{(k)}+D_{\mu}^{\alpha \beta}(\mathcal{B}) \frac{\delta}{\delta \mathcal{A}_{m \mid \mu}^{* \beta}} \tilde{\Gamma}^{(k)}, \\
& \left.\frac{\delta}{\delta \mathcal{B}_{\mu}^{\alpha}} \Gamma^{(k)}=\frac{\delta}{\delta \mathcal{B}_{\mu}^{\alpha}} \tilde{\Gamma}^{(k)}-g f^{\alpha \beta \gamma} \bar{C}_{m \mid}^{\beta} \frac{\delta}{\delta \mathcal{A}_{m \mid \mu}^{* \gamma}} \tilde{\Gamma}^{(k)}, \quad k=1,2.21\right)
\end{aligned}
$$

Then, from the Eqs. (4.11), (4.18), (4.20), (4.21), we find that

$$
\frac{\delta}{\delta \bar{C}_{m \mid}^{\alpha}} \tilde{\Gamma}^{(k)}=0
$$

the functionals $\tilde{\Gamma}^{(k)}, k=1,2$ do not depend on the fields $\bar{C}_{m \mid}^{\alpha}$,

$\tilde{\Gamma}^{(k)}=\tilde{\Gamma}^{(k)}\left(\Omega_{m \mid}, \Omega_{m \mid}^{*}, \mathcal{B}, \xi, \theta\right)$.

Here and below we will use the notations

$$
\begin{aligned}
& \Omega_{m \mid}=\left\{\mathcal{A}_{m \mid}, \psi_{m \mid}, \bar{\psi}_{m \mid}, \varphi_{m \mid}, C_{m \mid}\right\}, \\
& \Omega_{m \mid}^{*}=\left\{\mathcal{A}_{m \mid}^{*}, \psi^{*}, \bar{\psi}^{*}, \varphi^{*}, C^{*}\right\} .
\end{aligned}
$$

Now, thanks to (4.18), (4.24), (4.25), the equations (4.12) and (4.13) rewrite as

$$
\begin{aligned}
& \frac{1}{2}\left(\tilde{\Gamma}^{(1)}, \tilde{\Gamma}^{(1)}\right)-\int d x\left(\theta \frac{\delta}{\delta \mathcal{B}}\right) \tilde{\Gamma}^{(1)}=0, \\
& 2 \xi \frac{\partial}{\partial \xi} \tilde{\Gamma}^{(1)}=\left(\tilde{\Gamma}^{(1)}, \tilde{\Gamma}^{(2)}\right) \\
& \quad+\int d x\left(\Omega_{m \mid}^{*} \frac{\delta}{\delta \Omega_{m \mid}^{*}}-\Omega_{m \mid} \frac{\delta}{\delta \Omega_{m \mid}}\right) \tilde{\Gamma}^{(1)} \\
& \quad-\int d x\left(\theta \frac{\delta}{\delta \mathcal{B}}\right) \tilde{\Gamma}^{(2)},
\end{aligned}
$$

where the notation for antibracket $[3,4]$

$$
(F, G)=\frac{1}{2} F \int d x\left(\frac{\overleftarrow{\delta}}{\delta \Omega_{m \mid}} \frac{\delta}{\delta \Omega_{m \mid}^{*}}-\frac{\overleftarrow{\delta}}{\delta \Omega_{m \mid}^{*}} \frac{\delta}{\delta \Omega_{m \mid}}\right) G
$$

is used. Further, taking into account (4.18), (4.24) and

$$
\begin{aligned}
\Gamma^{(k)} & \int d x\left[\frac{\overleftarrow{\delta}}{\delta \mathcal{B}_{\mu}^{\beta}} D_{\mu}^{\beta \alpha}(\mathcal{B})+g \varepsilon^{\beta \gamma \alpha}\right. \\
& \left.\times\left(\frac{\overleftarrow{\delta}}{\delta \bar{C}_{m \mid}^{\beta}} \bar{C}_{m \mid}^{\gamma}+\frac{\overleftarrow{\delta}}{\delta A_{\mu}^{* \beta}} A_{\mu}^{* \gamma}\right)\right] \\
= & \tilde{\Gamma}^{(k)} \int d x\left[\frac{\overleftarrow{\delta}}{\delta \mathcal{B}_{\mu}^{\beta}} D_{\mu}^{\beta \alpha}(\mathcal{B})+g \varepsilon^{\beta \gamma \alpha} \frac{\overleftarrow{\delta}}{\delta \mathcal{A}_{m \mid \mu}^{* \beta}} \mathcal{A}_{m \mid \mu}^{* \gamma}\right]
\end{aligned}
$$

we find that

$\Gamma^{(k)} \overleftarrow{h_{m \mid}^{\alpha}} \omega_{\alpha}=\tilde{\Gamma}^{(k)} \overleftarrow{\hat{h}_{m \mid}^{\alpha}} \omega_{\alpha}=0, k=1,2$

where the operator $\overleftarrow{\tilde{h}_{m \mid}^{\alpha}} \omega_{\alpha}$ is defined in the equality (3.13) with the substitution $\Omega \rightarrow \Omega_{m \mid}, \Omega^{*} \rightarrow \Omega_{m \mid}^{*}$.

Later, on when studying the tensor structure of divergent parts of the generating functional of vertex functions, it is useful to use the consequence of the Eq. (4.30) corresponding to the particular case of $T_{m \mid}$-symmetry when $\omega_{\alpha}(x)=$ const,

$\tilde{\Gamma}^{(k)} \overleftarrow{T_{m \mid}^{\alpha}}=0, k=1,2$

where the operators $\overleftarrow{T_{m}^{\alpha}}$ are defined by the equalities (3.15) with substitution $\Omega \rightarrow \Omega_{m \mid}, \Omega^{*} \rightarrow \Omega_{m \mid}^{*}$.

\section{Multiplicative renormalization}

In this section, we study a structure of renormalization in the model under consideration, and find multiplicative character of renormalizability. The main role is played by solving the extended master-equation (3.10) and the equation describing the gauge dependence (3.18). We will show that the renormalized quantum action and the effective action satisfy this 
equation exactly to every order of loop expansions. The structure of the renormalized action is determined by the same monomials in fields and antifields as the non-renormalized quantum action does but with constants defined by the divergences of the effective action. For simplicity of notations, we will often omit the lower index $m \mid$ in arguments of functional $\Gamma$.

\subsection{Tree approximation $(\eta=0)$}

Consider the tree approximation for the functional $\Gamma, \Gamma_{0}=$ $S_{\text {ext }}$, which in new variables reads

$\Gamma_{0}=\Gamma_{0 \mid 0}+\Gamma_{0}^{(1)}+\chi \Gamma_{0}^{(2)}$,

$\Gamma_{0}^{(1)}=\tilde{\Gamma}_{0}^{(1)}, \quad \Gamma_{0}^{(2)}=\tilde{\Gamma}_{0}^{(2)}$,

Represent the functional $\tilde{\Gamma}_{0}^{(1)}$ in the form

$\tilde{\Gamma}_{0}^{(1)}=\Gamma_{0 \theta}+\Gamma_{0 \Omega^{*}}+\Gamma_{0 \psi}+\Gamma_{0 \varphi}+\Gamma_{0 \psi \varphi}+\Gamma_{0 \mathcal{A B}}$,

$\Gamma_{0 \Omega^{*}}=\Gamma_{0 \mathcal{A}^{*}}+\Gamma_{0 C^{*}}+\Gamma_{0 \psi^{*}}+\Gamma_{0 \bar{\psi}^{*}}+\Gamma_{0 \varphi^{*}}$,

where the following notations useful for further calculations will be used,

$$
\begin{aligned}
\Gamma_{0 \theta}= & \Gamma_{0 \mid 6}, \quad \Gamma_{0 \mathcal{A}^{*}}=\Gamma_{0 \mid 7}+\Gamma_{0 \mid 8}, \quad \Gamma_{0 C^{*}}=\Gamma_{0 \mid 9}, \\
\Gamma_{0 \psi^{*}}= & \Gamma_{0 \mid 10}, \quad \Gamma_{0 \bar{\psi}^{*}}=\Gamma_{0 \mid 11}, \quad \Gamma_{0 \varphi^{*}}=\Gamma_{0 \mid 12} \\
\Gamma_{0 \psi}= & \Gamma_{0 \mid 13}+\Gamma_{0 \mid 14}+\Gamma_{0 \mid 15} \\
\Gamma_{0 \varphi}= & \Gamma_{0 \mid 16}+\Gamma_{0 \mid 17}+\Gamma_{0 \mid 18}+\Gamma_{0 \mid 19}+\Gamma_{0 \mid 20} \\
& \Gamma_{0 \psi \varphi}=\Gamma_{0 \mid 21}, \\
\Gamma_{0 \mathcal{A B}}= & \Gamma_{0 \mid 22}
\end{aligned}
$$

The functional $\tilde{\Gamma}_{0}^{(2)}$ has the form

$\tilde{\Gamma}_{0}^{(2)}=\Gamma_{0 \mid 1}+\Gamma_{0 \mid 2}+\Gamma_{0 \mid 3}++\Gamma_{0 \mid 4}+\Gamma_{0 \mid 5}$.

Remind that the functional $\Gamma_{0}$ satisfies the Eq. (2.20)(2.24).

\section{$5.2(1+1)$-loop approximation (order $\eta^{l+1}$ )}

The proof of multiplicative renormalizability will be given by the method of mathematical induction in loop expansions of the effective action applying the scheme of minimal subtractions. To this end, let us suppose we have found the parameters $Z_{k_{\text {ind }}}$,

$$
\begin{aligned}
Z_{k_{\text {ind }}}= & Z_{k_{\text {ind }}}^{[l]}+O\left(\eta^{l+1}\right), \\
Z_{k_{\text {ind }}}^{[l]}= & 1+\sum_{n=1}^{l} \eta^{n} z_{k_{\text {ind }}, n}, \quad \forall k_{\text {ind }}, \\
& \dot{z}_{k_{\text {con }}, n}=0, \quad \forall k_{\text {con }}, \quad 1 \leq n \leq l,
\end{aligned}
$$

so that the $l$-loop approximation for $\Gamma, \Gamma^{[l]}=\sum_{n=0}^{l} \eta^{n} \Gamma_{n}$, is a finite functional. We will show that the $(l+1)$-loop approximation for $Z_{k_{i n d}}$ can be picked up so that,

$$
\begin{aligned}
Z_{k_{\text {ind }}} & =Z_{k_{\text {ind }}}^{[l+1]}+O\left(\eta^{l+2}\right), \\
Z_{k_{\text {ind }}}^{[l+1]} & =Z_{k_{\text {ind }}}^{[l]}+z_{k_{\text {ind }}, l+1},
\end{aligned}
$$

which compensates divergences of the $(l+1)$-loop approximation of the functional $\Gamma$.

Represent the action $S_{R}$ in the form

$S_{R}=S_{R}^{[l]}+\eta^{l+1} s_{l+1}+O\left(\eta^{l+2}\right)$,

where $S_{R}^{[l]}$ is the action $S_{R}$ with independent parameters $Z_{k_{\text {ind }}}$ replaced by $Z_{k_{\text {ind }}}^{[l]}$ satisfying the Eqs. (2.20)-(2.24) and functional $s_{l+1}$ is equal to

$s_{l+1}=s_{l+1}^{(1)}+\chi s_{l+1}^{(2)}$.

In this section we will use the short notations for variational derivatives of the type

$\frac{\delta}{\delta \mathcal{A}} \rightarrow \partial_{\mathcal{A}}, \quad \mathcal{A} \partial_{\mathcal{A}}=\int d x \mathcal{A} \frac{\delta}{\delta \mathcal{A}}$,

when it will not lead to uncertainties.

Now let us study the structure of the functional $\Gamma$ with taking into account the $(l+1)$-loop approximation. It describes by diagrams with vertices of the action $S_{R}$ with parameters $z_{k_{\text {ind }}, n}, 0 \leq n \leq l+1, z_{k_{\text {ind }}, 0}=0$, i.e. with vertices of action $S_{R}^{[l]}$ and with vertices of $s_{l+1}$. Because we are interested in diagrams of loop order not higher than $l+1$, the vertices from $s_{l+1}$ cannot appear in loop diagrams, i.e. the vertices from $s_{l+1}$ give "tree" contribution to $\Gamma$ equal to $\eta^{l+1} s_{l+1}$. The rest diagrams are generated by the action $S_{R}^{[l]}$. Denote the contribution of these diagrams to functional $\Gamma$ as $\Gamma\left(S_{R}^{[l]}\right)$, i.e.,

$\Gamma=\Gamma\left(S_{R}^{[l]}\right)+\eta^{l+1} s_{l+1}+O\left(\eta^{l+2}\right)$.

Because the action $S_{R}^{[l]}$ satisfies the Eqs. (2.20)-(2.24), the functional $\Gamma\left(S_{R}^{[l]}\right)$ satisfies these equations too (with substitutions $\left.Q, \bar{C}, B \rightarrow Q_{m \mid}, \bar{C}_{m \mid}, B_{m \mid}\right)$.

Represent the functional $\Gamma\left(S_{R}^{[l]}\right)$ in the form

$\Gamma\left(S_{R}^{[l]}\right)=\Gamma_{0 \mid 0}+\Gamma^{(1)}\left(S_{R}^{[l]}\right)+\chi \Gamma^{(2)}\left(S_{R}^{[l]}\right)$,

Repeating calculations made in Sect. 3 we find that

$$
\begin{aligned}
& \Gamma^{(k)}\left(S_{R}^{[l]} \mid Q_{m \mid}, \bar{C}_{m \mid}, \mathcal{B}, Q^{*}, B_{m \mid}, \xi, \theta\right) \\
& \quad=\tilde{\Gamma}^{(k)}\left(S_{R}^{[l]} \mid \Omega_{m \mid}, \Omega_{m \mid}^{*}, \mathcal{B}, \xi, \theta\right), k=1,2,
\end{aligned}
$$

and functionals $\tilde{\Gamma}^{(k)}\left(S_{R}^{[l]}\right)$ satisfy the Eqs. (4.26), (4.27) and (4.30).

Represent the functionals $\tilde{\Gamma}^{(k)}\left(S_{R}^{[l]}\right)$ in the sum of divergent and finite (after removing a regularization) terms. Taking into account that the functionals $\tilde{\Gamma}^{(k)}\left(S_{R}^{[l]}\right)$ are finite to $n$-loop 
approximations, $0 \leq n \leq l$, by proposition, we obtain

$$
\begin{aligned}
& \tilde{\Gamma}^{(k)}\left(S_{R}^{[l]}\right)=\tilde{\Gamma}^{(k)}\left(S_{R}^{[l]}\right)_{\text {fin }} \\
& \quad+\eta^{l+1} \tilde{\Gamma}^{(k)}\left(S_{R}^{[l]}\right)_{l+1, \text { div }}+O\left(\eta^{l+2}\right) .
\end{aligned}
$$

To simplify the presentations, we introduce the notations

$$
\begin{aligned}
& \tilde{\Gamma}^{(k)}\left(S_{R}^{[l]}\right)_{l+1, \mathrm{div}}=u_{l+1}^{(k)}, \\
& \quad u_{l+1}^{(1)}+\chi u_{l+1}^{(2)}=u_{l+1}, k=1,2 .
\end{aligned}
$$

Then,

$\Gamma=\Gamma\left(S_{R}^{[l]}\right)_{\text {fin }}+\eta^{l+1}\left(u_{l+1}+s_{l+1}\right)+O\left(\eta^{l+2}\right)$,

and functionals $u_{l+1}^{(k)}$ are local polynomials of their arguments with quantum numbers of the action $S_{\text {ext }}$ and contain only divergent summands (formalism of minimal subtraction). Then, they satisfy the equations which follow from the Eqs. (4.26), (4.27) and (4.30),

$$
\begin{aligned}
& \left(\tilde{\Gamma}_{0}^{(1)}, u_{l+1}^{(1)}\right)-\int d x\left(\theta \frac{\delta}{\delta \mathcal{B}}\right) u_{l+1}^{(1)}=0 \\
& 2 \xi \frac{\partial}{\partial \xi} u_{l+1}^{(1)}=\left(\tilde{\Gamma}_{0}^{(1)}, u_{l+1}^{(2)}\right)-\left(\tilde{\Gamma}_{0}^{(2)}, u_{l+1}^{(1)}\right) \\
& \quad-\int d x\left(\theta \frac{\delta}{\delta \mathcal{B}}\right) u_{l+1}^{(2)} \\
& \quad+\int d x\left(\Omega_{m \mid}^{*} \frac{\delta}{\delta \Omega_{m \mid}^{*}}-\Omega_{m \mid} \frac{\delta}{\delta \Omega_{m \mid}}\right) u_{l+1}^{(1)} \\
& u_{l+1}^{(k)} \overleftarrow{h^{\alpha}} \omega_{\alpha}=0, \\
& u_{l+1}^{(k)} \overleftarrow{T^{\alpha}}=0, \quad k=1,2 .
\end{aligned}
$$

Notice that the form of Eqs. (5.22)-(5.25) does not depend on index $l$.

Taking into account the quantum numbers, the axial-, Poincare- and T-symmetries we find general expression for local functional $u_{l+1}^{(2)}$,

$u_{l+1}^{(2)}=\sum_{k=1}^{5} q_{k, l+1} \Gamma_{0 \mid k}+q_{1, l+1}^{\prime} \mathcal{A}^{*} \mathcal{B}$,

where $q_{k, l+1}, k=1, \ldots, 5$, and $q_{1, l+1}^{\prime}$ are arbitrary constants. Further, using the Eq. (5.24) for $u_{l+1}^{(2)}$, we find $q_{1, l+1}^{\prime}=$ 0 . Final expression for $q_{1, l+1}^{\prime}=0$ reads

$u_{l+1}^{(2)}=\sum_{k=1}^{5} q_{k, l+1} \Gamma_{0 \mid k}$.

Notice that the functional $u_{l+1}^{(2)}$ does not depend on the fields $\theta$ and $\mathcal{B}$.

Due to the expression (5.27) for $u_{l+1}^{(2)}$, the Eq. (5.23) reduces to

$2 \xi \frac{\partial}{\partial \xi} u_{l+1}^{(1)}=\hat{L}\left(1+q_{, l+1}\right) \tilde{\Gamma}_{0}^{(1)}$, where the operator $\hat{L}$ is defined in (3.18) with substitution $Z_{k} \rightarrow 1+q_{k, l+1}, k=1, \ldots, 5$.

\subsubsection{Solution to equation (5.22)}

Consider a solution to the Eq. (5.22) for the functional $\tilde{\Gamma}_{l+1, \mathrm{div}}^{(1)}=\tilde{\Gamma}\left(S_{R}^{[l]}\right)_{l+1, \mathrm{div}}^{(1)}=u_{l+1}^{(1)}$ using the presentation

$$
\begin{aligned}
u_{l+1}^{(1)}= & M_{\theta, l+1}+M_{\Omega^{*}, l+1}+M_{\psi, l+1}+M_{\varphi, l+1} \\
& +M_{\psi \varphi, l+1}+M_{\mathcal{A B}, l+1}(\mathcal{A}, \mathcal{B}), \\
M_{\Omega^{*}, l+1}= & M_{\mathcal{A}^{*}, l+1}+M_{C^{*}, l+1}+M_{\psi^{*}, l+1} \\
& +M_{\bar{\psi}^{*}, l+1}+M_{\varphi^{*}, l+1}
\end{aligned}
$$

To this end, we will find the general form of the functional $\tilde{\Gamma}_{l+1 \text {,div }}^{(1)}$, following from locality, quantum numbers, axial-, Poincare-, $T$ - symmetries and, partially, of the gauge symmetry with respect to the external field $\mathcal{B}$. In fact, all needed calculations copy calculations made in Sect. 3 when constructing the general form of the functional $\tilde{P}^{(1)}$ (see formulas (3.21)-(3.30)) with evident substitutions like $\tilde{P}_{\theta}^{(1)} \rightarrow M_{\theta}$. Here we give the final results only. The functional $M_{\theta, l+1}$ reads

$M_{\theta, l+1}=q_{6, l+1} \Gamma_{0 \mid 6}$.

For the functionals linear in antifields we find

$$
\begin{aligned}
& M_{\mathcal{A}^{*}, l+1}=q_{7, l+1} \Gamma_{0 \mid 7}+q_{8, l+1} \Gamma_{0 \mid 8}, \\
& M_{C^{*}, l+1}=q_{9, l+1} \Gamma_{0 \mid 9}, \\
& M_{\psi^{*}, l+1}=q_{10, l+1} \Gamma_{0 \mid 10}, \quad M_{\bar{\psi}^{*}, l+1} \\
& \quad=q_{11, l+1} \Gamma_{0 \mid 11}, \quad M_{\varphi^{*}, l+1}=q_{12, l+1} \Gamma_{0 \mid 12} .
\end{aligned}
$$

For the functionals $M_{\psi, l+1}, M_{\varphi, l+1}, M_{\psi \varphi, l+1}$, we obtain

$$
\begin{aligned}
M_{\psi, l+1}= & q_{13, l+1} \Gamma_{0 \mid 13}+q_{14, l+1} \Gamma_{0 \mid 14}+q_{15, l+1} \Gamma_{0 \mid 15} \\
M_{\varphi, l+1}= & q_{16, l+1} \Gamma_{0 \mid 16}+q_{17, l+1} \Gamma_{0 \mid 17} \\
& +M_{\varphi 18, l+1}+q_{19, l+1} \Gamma_{0 \mid 1}+q_{20, l+1} \Gamma_{0 \mid 20}
\end{aligned}
$$

$$
M_{\psi \varphi, l+1}=q_{21, l+1} \Gamma_{0 \mid 21},
$$$$
M_{\varphi 18, l+1}=\int d x\left[\frac{g^{2}}{2} q_{18}^{\alpha \beta \gamma \sigma} \mathcal{A}_{\mu}^{\alpha} \varphi^{\beta} \mathcal{A}_{\mu}^{\gamma} \varphi^{\sigma}\right],
$$$$
q_{18}^{\alpha \beta \gamma \sigma}=q_{18}^{\gamma \beta \alpha \sigma}=q_{18}^{\alpha \sigma \gamma \beta} \text {. }
$$ 
Consider the consequences of the equality to zero for contributions proportional to $\theta$.

$$
\begin{aligned}
& \theta \bar{\psi} \psi \Rightarrow\left(q_{6, l+1}+q_{14, l+1}\right) \partial_{\mathcal{A}_{\mu}^{\alpha}} \\
& \times \Gamma_{0 \mid 14}-q_{13, l+1} \partial_{\mathcal{B}_{\mu}^{\alpha}} \Gamma_{0 \mid 13}=0 \\
& \Rightarrow q_{14, l+1}=q_{13, l+1}-q_{6, l+1} \text {; } \\
& \theta \mathcal{A}^{*} C \Rightarrow\left(q_{6, l+1}+q_{8, l+1}\right) \partial_{\mathcal{A}_{\mu}^{\alpha}} \\
& \times \Gamma_{0 \mid 8}-q_{7, l+1} \partial_{\mathcal{B}_{\mu}^{\alpha}} \Gamma_{0 \mid 7}=0 \\
& \Rightarrow q_{8, l+1}=q_{7, l+1}-q_{6, l+1} \text {; } \\
& \theta\left(D_{\varphi}(\mathcal{B}) \varphi\right) \varphi \Rightarrow\left(q_{6, l+1}+q_{17, l+1}\right) \partial_{\mathcal{A}_{\mu}^{\alpha}} \\
& \times \Gamma_{0 \mid 17}-q_{16, l+1} \partial_{\mathcal{B}_{\mu}^{\alpha}} \Gamma_{0 \mid 16}=0 \\
& \Rightarrow q_{17, l+1}=q_{16, l+1}-q_{6, l+1} \text {; } \\
& \theta \mathcal{A} \varphi \varphi \Rightarrow q_{6, l+1} \partial_{\mathcal{A}_{\mu}^{\alpha}} \\
& \times \Gamma_{0 \mid 18}+\partial_{\mathcal{A}_{\mu}^{\alpha}} M_{\varphi 18, l+1}-q_{17, l+1} \partial_{\mathcal{B}_{\mu}^{\alpha}} \Gamma_{0 \mid 17}=0 \\
& \Rightarrow M_{\varphi 18, l+1} \\
& =q_{18, l+1} \Gamma_{0 \mid 18}, \quad q_{18, l+1}=q_{16, l+1}-2 q_{6, l+1} ; \\
& \theta \mathcal{A}^{n} \mathcal{B}^{m} \Rightarrow q_{6, l+1} \partial_{\mathcal{A}_{\mu}^{\alpha}} \\
& \times \Gamma_{0 \mid 22}(V)+\partial_{\mathcal{A}_{\mu}^{\alpha}} M_{\mathcal{A B}, l+1}-\partial_{\mathcal{B}_{\mu}^{\alpha}} M_{\mathcal{A B}, l+1}=0 \\
& \Rightarrow M_{\mathcal{A B}, l+1}=-q_{6, l+1} \mathcal{A}_{\mathcal{A}} \Gamma_{0 \mid 22}(V) \\
& +M_{\mathcal{A B}, l+1}^{\prime}(V) \text {, }
\end{aligned}
$$

where $V=\mathcal{A}+\mathcal{B}$ and $M_{\mathcal{A B}, l+1}^{\prime}(V)$ is an arbitrary functional of $V$.

When $\theta=0$, the Eq. (5.22) reduces to

$$
\begin{aligned}
\int & d x\left(\Gamma_{0 \Omega^{*}}+\Gamma_{0 \psi}+\Gamma_{0 \varphi}+\Gamma_{0 \psi \varphi}+\Gamma_{0 \mathcal{A B}}\right) \\
& \times\left(\overleftarrow{\partial}_{\Omega} \partial_{\Omega^{*}}-\overleftarrow{\partial}_{\Omega^{*}} \partial_{\Omega}\right) \\
& \times\left(M_{\Omega^{*}, l+1}+M_{\psi, l+1}+M_{\varphi, l+1}\right. \\
& +M_{\psi \varphi, l+1}+M_{\mathcal{A B}, l+1}=0
\end{aligned}
$$

which is not more than linear in antifields.

We have

$$
\begin{gathered}
\mathcal{A}^{*} \mathcal{A} C C \Rightarrow 2 q_{8, l+1} \Gamma_{0 \mid 8} \frac{\overleftarrow{\delta}}{\delta \mathcal{A}_{\mu}^{\alpha}} \frac{\delta}{\delta \mathcal{A}_{\mu}^{* \alpha}} \\
\times \Gamma_{0 \mid 8}+\left(q_{8, l+1}+q_{9, l+1}\right) \Gamma_{0 \mid 8} \frac{\overleftarrow{\delta}}{\delta C^{\alpha}} \frac{\delta}{\delta C^{* \alpha}} \Gamma_{0 \mid 9}=0 \\
\Rightarrow q_{9, l+1}=q_{8, l+1}=q_{7, l+1}-q_{6, l+1} ; \\
\psi^{*} \psi C C \Rightarrow\left(q_{9, l+1}+q_{10, l+1}\right) \Gamma_{0 \mid 10} \frac{\overleftarrow{\delta}}{\delta C^{\alpha}} \frac{\delta}{\delta C^{* \alpha}} \\
\times \Gamma_{0 \mid 9}+2 q_{10, l+1} \Gamma_{0 \mid 10} \frac{\overleftarrow{\delta}}{\delta \psi_{j}} \frac{\delta}{\delta \psi_{j}^{*}} \Gamma_{0 \mid 10}=0 \\
\Rightarrow q_{10, l+1}=q_{9, l+1}=q_{7, l+1}-q_{6, l+1} \\
\overline{\psi \psi}^{*} C C \Rightarrow\left(q_{9, l+1}+q_{11, l+1}\right) \Gamma_{0 \mid 11} \frac{\overleftarrow{\delta}}{\delta C^{\alpha}} \frac{\delta}{\delta C^{* \alpha}}
\end{gathered}
$$

$$
\begin{gathered}
\times \Gamma_{0 \mid 9}+2 q_{11, l+1} \Gamma_{0 \mid 11} \frac{\overleftarrow{\delta}}{\delta \bar{\psi}_{j}} \frac{\delta}{\delta \bar{\psi}_{j}^{*}} \Gamma_{0 \mid 10}=0 \\
\Rightarrow q_{11, l+1}=q_{9, l+1}=q_{7, l+1}-q_{6, l+1} \\
\bar{\psi} \psi \varphi C \Rightarrow\left(q_{12, l+1}+q_{21, l+1}\right) \Gamma_{0 \mid 21} \frac{\overleftarrow{\delta}}{\delta \varphi^{\alpha}} \frac{\delta}{\delta \varphi^{* \alpha}} \\
\times \Gamma_{0 \mid 12}+\left(q_{10, l+1}+q_{21, l+1}\right) \Gamma_{0 \mid 21} \frac{\overleftarrow{\delta}}{\delta \psi_{j}} \frac{\delta}{\delta \psi_{j}^{*}} \Gamma_{0 \mid 10} \\
+\left(q_{11, l+1}+q_{21, l+1}\right) \Gamma_{0 \mid 21} \frac{\overleftarrow{\delta}}{\delta \bar{\psi}_{j}} \frac{\delta}{\delta \bar{\psi}_{j}^{*}} \Gamma_{0 \mid 11}=0 \Rightarrow \\
\times q_{12, l+1}=q_{10, l+1}=q_{7, l+1}-q_{6, l+1}
\end{gathered}
$$

where the relation $q_{11, l+1}=q_{10, l+1}$ was used.

$$
\begin{aligned}
& \mathcal{A}^{n} \mathcal{B}^{k} C \Rightarrow \Gamma_{0 \mid 22} \frac{\overleftarrow{\delta}}{\delta \mathcal{A}_{\mu}^{\alpha}} \frac{\delta}{\delta \mathcal{A}_{\mu}^{* \alpha}}\left(q_{7, l+1} \Gamma_{0 \mid 7}+q_{8, l+1} \Gamma_{0 \mid 8}\right) \\
& \quad-\left(\Gamma_{0 \mid 7}+\Gamma_{0 \mid 8}\right) \frac{\overleftarrow{\delta}}{\delta \mathcal{A}_{\mu}^{* \alpha}} \frac{\delta}{\delta \mathcal{A}_{\mu}^{\alpha}} M_{\mathcal{A B}, l+1}=0 \Rightarrow \\
& M_{\mathcal{A B}, l+1}^{\prime}=q_{22, l+1} \Gamma_{0 \mid 22}(V), \quad M_{\mathcal{A B}, l+1} \\
& \quad=-q_{6, l+1} \mathcal{A}_{\mathcal{A}} \Gamma_{0 \mid 22}(V)+q_{22, l+1} \Gamma_{0 \mid 22}(V)
\end{aligned}
$$

Thus, the divergences $u_{l+1}^{(1)}$ can be represented in the form

$u_{l+1}^{(1)}=\sum_{k=6}^{21} q_{k, l+1} \Gamma_{0 \mid k}+M_{\mathcal{A B}, l+1}$,

and the functional $M_{\mathcal{A B}, l+1}$ is given by the formula (5.48). As it was noted above, all functionals considered as polynomials are independent, and polynomials $\Gamma_{0 \mid 6}-\Gamma_{0 \mid 21}$ are homogeneous with respect to the fields $\Omega$ and the antifields $\Omega^{*}$.

\subsubsection{Solution to equation (5.28)}

Taking into consideration that the polynomials $\Gamma_{0 \mid k}, k=$ $6, \ldots, 21$, are eigen for operator $\hat{L}$, we find that Eq. (5.28) is reduced to the set of equations

$2 \xi \frac{\partial}{\partial \xi} q_{k, l+1}=\varrho_{k}, k=6, \ldots, 21$,

$2 \xi \frac{\partial}{\partial \xi} M_{\mathcal{A B}, l+1}=q_{1, l+1} \mathcal{A} \partial_{\mathcal{A}} \Gamma_{0 \mid 22}(V)$,

where $\varrho_{k}$ are the eigenvalues of the operator $\hat{L}$. 
We have

$$
\begin{aligned}
k= & \Rightarrow 2 \xi \dot{q}_{6, l+1}=-q_{1, l+1} \\
& \Rightarrow q_{1, l+1}=-2 \xi \dot{q}_{6, l+1} \\
k= & \Rightarrow 2 \xi \dot{q}_{7, l+1}=-q_{1, l+1}+q_{2, l+1} \\
& \Rightarrow q_{2, l+1}=2 \xi \dot{q}_{7, l+1}-2 \xi \dot{q}_{6, l+1} \\
k= & 13 \Rightarrow 2 \xi \dot{q}_{13, l+1}=q_{3, l+1}+q_{4, l+1} \\
& \Rightarrow q_{4, l+1}=2 \xi \dot{q}_{13, l+1}-q_{3, l+1} \\
k= & \Rightarrow 2 \xi \dot{q}_{15, l+1}=q_{3, l+1}+q_{4, l+1} \\
& \Rightarrow \dot{q}_{23, l+1}=0, q_{15, l+1}=q_{13, l+1}+q_{23, l+1} \\
k= & 16 \Rightarrow 2 \xi \dot{q}_{16, l+1}=2 q_{5, l+1} \\
& \Rightarrow q_{5, l+1}=\xi \dot{q}_{16, l+1} \\
k= & 19 \Rightarrow 2 \xi \dot{q}_{19, l+1}=2 q_{5, l+1} \\
& \Rightarrow \dot{q}_{24, l+1}=0, q_{19, l+1}=q_{16, l+1}+q_{24, l+1} \\
k= & \Rightarrow 2 \xi \dot{q}_{20, l+1}=4 q_{5, l+1} \\
& \Rightarrow \dot{q}_{25, l+1}=0, q_{20, l+1}=2 q_{16, l+1}+q_{25, l+1} \\
k=21 & \Rightarrow 2 \xi \dot{q}_{21, l+1}=q_{3, l+1}+q_{4, l+1}+q_{5, l+1} \\
& \Rightarrow \dot{q}_{26, l+1}=0, q_{21, l+1}=q_{13, l+1} \\
& +\frac{1}{2} q_{16, l+1}+q_{26, l+1}
\end{aligned}
$$

Finally, from (5.51) it follows

$$
\begin{aligned}
& 2 \xi \dot{q}_{22, l+1} \Gamma_{0 \mid 22}-\left(2 \xi \dot{q}_{6, l+1}+q_{1, l+1}\right) \mathcal{A}_{\mathcal{A}} \Gamma_{0 \mid 22}(V)=0 \\
& \quad \Rightarrow 2 \xi \dot{q}_{22, l+1} \Gamma_{0 \mid 22}=0 \Rightarrow \dot{q}_{22, l+1}=0,
\end{aligned}
$$

where we used the result of the block $k=6$.

Finally, the solution to the set of Eqs. (5.22)-(5.25) for $u_{l+1}^{(k)}, k=1,2$, can be represented in the form

$$
\begin{aligned}
u_{l+1}^{(1)}= & \sum_{k=6}^{21} q_{k, l+1} \Gamma_{0 \mid k}-q_{6, l+1} \mathcal{A}_{\mathcal{A}} \Gamma_{0 \mid 22}(V) \\
& +q_{22, l+1} \Gamma_{0 \mid 22}(V), \\
u_{l+1}^{(2)}= & \sum_{k=1}^{5} q_{k, l+1} \Gamma_{0 \mid k} .
\end{aligned}
$$

Let $\left\{k_{\text {ind }}\right\}=\left\{\left\{K_{a}\right\}, 22\right\}, \quad\left\{K_{a}\right\}=\{3,6,7,13,16,23$, $\ldots, 26\}$. Then we have

$q_{k_{d e p}, l+1}=\sum_{a}\left(\left[X_{q \mid k_{d e p}, a}+Y_{q \mid k_{d e p}, a} \xi \partial \xi\right] q_{K_{a}, l+1}\right)$,

where the set $\left\{q_{k_{\text {dep }}, l+1}\right\}=\left\{\left\{q_{K_{a}, l+1}\right\}, q_{22}\right\}$ is the set of arbitrary numbers, 5 of them (with indexes $3,6,7,13,16$ ) can depend on $\xi$ and $\dot{q}_{22, l+1}=\dot{q}_{23, l+1}=\dot{q}_{24, l+1}=\dot{q}_{25, l+1}=$ $\dot{q}_{26, l+1}=0$. Numerical matrices $X_{q \mid k_{d e p}, a}$ and $Y_{q \mid k_{d e p}, a}$ in Eq. (5.63) can be restored by using the relations (5.38)(5.41), (5.44)-(5.47), (5.52)-(5.59).

\subsection{Finiteness of effective action}

Now, let us prove that one can choose the renormalization constants in such a way as to make the effective action finite to the $(l+1)$-loop approximation. To this end, we consider the divergent part of the effective action $\Gamma, \Gamma_{l+1 \text {,div }}$, described by the Eq. (5.21),

$\Gamma_{l+1, \operatorname{div}}=u_{l+1}+s_{l+1}=u_{l+1}^{(1)}+s_{l+1}^{(1)}+\chi\left[u_{l+1}^{(2)}+s_{l+1}^{(2)}\right]$.

It is easy to see that the functional $s_{l+1}$ satisfies the same Eqs. (5.22)-(5.25) as $u_{l+1}$ does (with substitution $u_{l+1}^{(k)} \rightarrow$ $\left.s_{l+1}^{(k)}, k=1,2\right)$, such that we have

$$
\begin{aligned}
s_{l+1}^{(1)}= & \sum_{k=6}^{21} z_{k, l+1} \Gamma_{0 \mid k}-z_{6, l+1} \mathcal{A} \partial_{\mathcal{A}} \\
& \times \Gamma_{0 \mid 22}(V)+z_{22, l+1} \Gamma_{0 \mid 22}(V), \\
s_{l+1}^{(2)}= & \sum_{k=1}^{5} z_{k, l+1} \Gamma_{0 \mid k}, \dot{z}_{22, l+1}=\dot{z}_{23, l+1} \\
= & \dot{z}_{24, l+1}=\dot{z}_{25, l+1}=\dot{z}_{26, l+1}=0, \\
z_{k_{d e p}, l+1}= & \sum_{a}\left(\left[X_{z \mid k_{d e p}, a}+Y_{z \mid k_{d e p}, a} \xi \partial_{\xi}\right] z_{K_{a}, l+1}\right), \\
X_{z \mid k_{d e p}, a}= & X_{q \mid k_{d e p}, a}, \quad Y_{z \mid k_{d e p}, a}=Y_{q \mid k_{d e p}, a} .
\end{aligned}
$$

Due to (5.61), (5.62), (5.65) and (5.66) the functional $\Gamma_{l+1, \operatorname{div}}$ (5.64) is written in the form

$$
\begin{aligned}
\Gamma_{l+1, \mathrm{div}}= & \sum_{k=6}^{21}\left(q_{k, l+1}+z_{k, l+1}\right) \Gamma_{0 \mid k} \\
& -\left(q_{6, l+1}+z_{6, l+1}\right) \mathcal{A}_{\mathcal{A}} \Gamma_{0 \mid 22}(V) \\
& +\left(q_{22, l+1}+z_{22, l+1}\right) \Gamma_{0 \mid 22}(V) \\
& +\chi \sum_{k=1}^{5}\left[\left(q_{k, l+1}+z_{k, l+1}\right) \Gamma_{0 \mid k}\right] .
\end{aligned}
$$

Now, let us take $z_{k_{\text {ind }}, l+1}$ as

$z_{k_{\text {ind }}, l+1}=-q_{k_{\text {ind }}, l+1}$

Then, due to (5.67), (3.49) and (5.63), we obtain that

$z_{k_{d e p}, l+1}=-q_{k_{d e p}, l+1}$

and as a consequence

$\Gamma_{l+1, \operatorname{div}}=0$.

Thus we have found the parameters $Z_{k_{\text {ind }}}$,

$$
\begin{aligned}
Z_{k_{\text {ind }}} & =Z_{k_{\text {ind }}}^{[l+1]}+O\left(\eta^{l+2}\right), \quad Z_{k_{\text {ind }}}^{[l+1]} \\
& =Z_{k_{\text {ind }}}^{[l]}+z_{k_{\text {ind }}, l+1}, \quad \dot{Z}_{k_{\text {con }}}^{[l+1]}=0
\end{aligned}
$$

so that the $(l+1)$-loop approximation for $\Gamma, \Gamma^{[l+1]}=$ $\sum_{n=0}^{l+1} \eta^{n} \Gamma_{n}$, is a finite functional. Note that the parameters 
$z_{k_{\text {ind }}, l+1}$ and $z_{k_{d e p}, l+1}$ given by Eqs. (5.70) and (5.67), respectively, are defined unambiguously by the divergences.

It is evident that this method works for any $l$, in particular for $l=0$, so that by using the loop induction method in Feynman diagrams for the functional $\Gamma$, we arrive at the following statement: for the $l$-loop approximation $\Gamma^{[l]}$ of the functional $\Gamma$ defined by the relations (4.2), (4.3) with arbitrary positive integer $l$,

$\Gamma^{[l]}=\sum_{n=0}^{l} \eta^{n} \Gamma_{n}$,

there exist the uniquely defined parameters $Z_{k_{i n d}}^{[l]}$, and parameters $z_{k_{d e p}}^{[l]}$ defining by Eq. (5.67),

$\dot{Z}_{k_{c o n}}^{[l]}=0$,

such that the functional $\Gamma^{[l]}$ does not contain divergences and $\Gamma$ satisfies the Eqs. (4.5)-(4.8).

\section{Gauge independence of physical parameters}

In that section, we find relations between some parameters of the action $S_{R}$ and the standard renormalization constants. Within the expression for $S_{R}$, we restrict ourselves only by desired vertexes in symbolic notation

$$
\begin{aligned}
S_{R}= & \int d x\left(Z_{22} Z_{6}^{-2} \partial A \partial A+g Z_{22} Z_{6}^{-3} A^{2} \partial A+Z_{13} \bar{\psi} \partial \psi\right. \\
& +m Z_{15} \bar{\psi} \psi+Z_{16} \partial \varphi \partial \varphi \\
& \left.+M^{2} Z_{19} \varphi^{2}+\lambda Z_{20} \varphi^{4}+\vartheta Z_{21} \varphi \bar{\psi} \psi \cdots\right)
\end{aligned}
$$

where the ellipsis means the rest vertexes. As the propagators of the fields $A, \psi$ and $\varphi$ are finite, they should be considered as renormalized fields. Then, we find:

$Z_{A}=Z_{22}^{1 / 2} Z_{6}^{-1}, \quad Z_{\psi}=Z_{13}^{1 / 2}, \quad Z_{\varphi}=Z_{16}^{1 / 2}$,

where $Z_{A}, Z_{\psi}$ and $Z_{\varphi}$ are the renormalization constants of the bare fields $A_{0}, \psi_{0}$ and $\varphi_{0}$. The coefficient of the second vertex in the expression (6.1) gives the renormalization for vertex $g A^{2} \partial A$,

$Z_{g A^{2} \partial A}=Z_{22} Z_{6}^{-3}$.

Analogously,

$$
\begin{aligned}
Z_{m \bar{\psi} \psi}= & Z_{15}, Z_{M^{2} \varphi^{2}}=Z_{19}, Z_{\lambda \varphi^{4}}=Z_{20}, \\
& Z_{\vartheta \varphi \bar{\psi} \psi}=Z_{21} .
\end{aligned}
$$

Then we find

$$
\begin{aligned}
g_{0} & =Z_{g} g, \quad Z_{g}=Z_{g A^{2} \partial A} Z_{A}^{-3}=Z_{22}^{-1 / 2}, \\
m_{0} & =Z_{m} m=Z_{m \bar{\psi} \psi} Z_{13}^{-1}=Z_{23}, \\
M_{0}^{2} & =Z_{M^{2}} M^{2}, \quad Z_{M^{2}}=Z_{19} Z_{16}^{-1}=Z_{24}, \\
\lambda_{0} & =Z_{\lambda} \lambda, \quad Z_{\lambda}=Z_{20} Z_{\varphi}^{-4}=Z_{25}, \\
\vartheta_{0} & =Z_{\vartheta} \vartheta, \quad Z_{\vartheta}=Z_{21} Z_{\varphi}^{-1 / 2} Z_{\psi}^{-1}=Z_{26} .
\end{aligned}
$$

It follows from the equations (5.75) that the renormalization constants of physical parameters $g, m, M^{2}, \lambda$ and $\vartheta$ do not depend on gauge,

$\partial_{\xi} Z_{g}=\partial_{\xi} Z_{m}=\partial_{\xi} Z_{M^{2}}=\partial_{\xi} Z_{\lambda}=\partial_{\xi} Z_{\vartheta}=0$.

\section{Summary}

In the present paper, within the background field formalism $[21,22,24]$, we have studied the renormalization and the gauge dependence of the $S U$ (2) Yang-Mills theory with the multiplets of massive spinor and scalar fields. The corresponding master-action of the BV-formalism $[3,4]$ has been extended with the help of additional fermion vector field $\theta$ and fermion constant parameter $\chi$. The action introduced is invariant under global supersymmetry and gauge transformations caused by the background vector field $\mathcal{B}$ appearing in the background field formalism. These symmetries allowed one to reduce, at the quantum level, the analysis of the renormalization and the gauge dependence problem for solutions to the extended master-equation and the gauge dependence equation. In comparison with our previous investigations of the multiplicative renormalization of the Yang-Mills theories [34], recent study involves the scalar fields which can be responsible for generating masses to physical particles through the mechanism of spontaneous symmetry breaking [36].

The proofs of multiplicative renormalizability and gauge independence of renormalization constants are based on the possibility to expand the extended effective action in loops, as well as to use the minimal subtraction scheme as to eliminate divergences. In addition, we propose the existence of a regularization preserving the used symmetries. Among the results obtained, we emphasize the rigorous proof of the gauge independence of all the physical parameters of the theory under consideration, to any order of loop expansions.

Acknowledgements We would like to thank Klaus Bering of Masaryk University for useful discussions and kindly help with References. Also we would like to thank anonymous Referee B who paid our attention to the paper [17]. P.M. Lavrov is grateful to the Physics Department of the Federal University of Juiz de Fora (MG, Brazil) for warm hospitality. The work of I.A. Batalin and I.V. Tyutin is supported in part by the RFBR Grant 17-02-00317. The work of P.M. Lavrov is supported partially by 
the Ministry of Education and Science of the Russian Federation, Grant 3.1386.2017 and by the RFBR Grant 18-02-00153.

Data Availability Statement This manuscript has no associated data or the data will not be deposited. [Authors' comment: This is a theoretical study and no experimental data has been listed.]

Open Access This article is distributed under the terms of the Creative Commons Attribution 4.0 International License (http://creativecomm ons.org/licenses/by/4.0/), which permits unrestricted use, distribution, and reproduction in any medium, provided you give appropriate credit to the original author(s) and the source, provide a link to the Creative Commons license, and indicate if changes were made. Funded by SCOAP ${ }^{3}$.

\section{References}

1. S. Weinberg, The Quantum Theory of Fields, vol. II (Cambridge University Press, Cambridge, 1996)

2. C.N. Yang, R.L. Mills, Considerations of isotopic spin and isotopic gauge invariance. Phys. Rev. 96, 191 (1954)

3. I.A. Batalin, G.A. Vilkovisky, Gauge algebra and quantization. Phys. Lett. B 102, 27 (1981)

4. I.A. Batalin, G.A. Vilkovisky, Quantization of gauge theories with linearly dependent generators. Phys. Rev. D 28, 2567 (1983)

5. C. Becchi, A. Rouet, R. Stora, The abelian Higgs Kibble Model, unitarity of the $S$-operator. Phys. Lett. B 52, 344 (1974)

6. I.V. Tyutin, Gauge invariance in field theory and statistical physics in operator formalism, LEBEDEV-75-39, pp 1-62 (1975). arXiv:0812.0580 [hep-th]

7. C. Becchi, A. Rouet, R. Stora, Renormalization of gauge theories. Ann. Phys. B 98, 287 (1976)

8. T. Kugo, I. Ojima, Local covariant operator formalism of nonabelian gauge theories and quark confinement problem. Progr. Theor. Phys. Suppl. 66, 1 (1979)

9. M. Henneaux, Hamiltonian form of the path integral for theories with a gauge freedom. Phys. Rep. 126, 1 (1985)

10. R.E. Kallosh, I.V. Tyutin, The equivalence theorem and gauge invariance in renormalizable theories. Sov. J. Nucl. Phys. 17, 98 (1973)

11. R. Jackiw, Functional evaluation of the effective potential. Phys. Rev. D 9, 1686 (1974)

12. L. Dolan, R. Jackiw, Gauge invariant signal for gauge symmetry breaking. Phys. Rev. D 9, 2904 (1974)

13. N.K. Nielsen, On the gauge dependence of spontaneous symmetry breaking in gauge theories. Nucl. Phys. B 101, 173 (1975)

14. R. Fukuda, T. Kugo, Gauge invariance in the effective action and potential. Phys. Rev. D 13, 3469 (1976)

15. P.M. Lavrov, I.V. Tyutin, On the structure of renormalization in gauge theories. Sov. J. Nucl. Phys. 34, 156 (1981)

16. P.M. Lavrov, I.V. Tyutin, On the generating functional for the vertex functions in Yang-Mills theories. Sov. J. Nucl. Phys. 34, 474 (1981)
17. O. Piguet, K. Sibold, Gauge independence in ordinary Yang-Mills theories. Nucl. Phys. B 253, 517 (1985)

18. H. Kluberg-Stern, J.B. Zuber, Renormalization of non-Abelian gauge theories in a background-field gauge. I. Green's functions. Phys. Rev. D 12, 482 (1975)

19. N.K. Nielsen, Removing the gauge parameter dependence of the effective potential by a field redefinition. Phys. Rev. D 90, 036008 (2014)

20. A.D. Plancencia, C. Tamarit, Convexity, gauge dependence and tunneling rates. JHEP 1610, 099 (2016)

21. B.S. De Witt, Quantum theory of gravity. II. The manifestly covariant theory. Phys. Rev. 162, 1195 (1967)

22. I.Ya. Arefeva, L.D. Faddeev, A.A. Slavnov, Generating functional for the s matrix in gauge theories. Theor. Math. Phys. 21, 1165 (1975)

23. I.Ya. Arefeva, L.D. Faddeev, A.A. Slavnov, Generating functional for the s matrix in gauge theories. Teor. Mat. Fiz. 21, 311-321 (1974)

24. L.F. Abbott, The background field method beyond one loop. Nucl. Phys. B 185, 189 (1981)

25. G. 't Hooft, An algorithm for the poles at dimension four in the dimensional regularization procedure. Nucl. Phys. B 62, 444 (1973)

26. M.T. Grisaru, P. van Nieuwenhuizen, C.C. Wu, Background field method versus normal field theory in explicit examples: one loop divergences in S matrix and Green's functions for Yang-Mills and gravitational fields. Phys. Rev. D 12, 3203 (1975)

27. D.M. Capper, A. MacLean, The background field method at two loops: a general gauge Yang-Mills calculation. Nucl. Phys. B 203, 413 (1982)

28. S. Ichinose, M. Omote, Renormalization using the backgroundfield formalism. Nucl. Phys. B 203, 221 (1982)

29. M.H. Goroff, A. Sagnotti, The ultraviolet behavior of Einstein gravity. Nucl. Phys. B 266, 709 (1986)

30. A.E.M. van de Ven, Two-loop quantum gravity. Nucl. Phys. B 378, 309 (1992)

31. P.A. Grassi, Algebraic renormalization of Yang-Mills theory with background field method. Nucl. Phys. B 462, 524 (1996)

32. A.O. Barvinsky, D. Blas, M. Herrero-Valea, S.M. Sibiryakov, C.F. Steinwachs, Renormalization of gauge theories in the backgroundfield approach. JHEP 1807, 035 (2018)

33. J. Frenkel, J.C. Taylor, Background gauge renormalization and BRST identities. Ann. Phys. 389, 234 (2018)

34. I.A. Batalin, P.M. Lavrov, I.V. Tyutin, Multiplicative renormalization of Yang-Mills theories in the background-field formalism. Eur. Phys. J. C 78, 570 (2018)

35. F.T. Brandt, J. Frenkel, D.G.C. McKeon, Renormalization of sixdimensional Yang-Mills theory in a background gauge field. Phys. Rev. D 99, 025003 (2019)

36. P.W. Higgs, Broken symmetries, massless particles and gauge fields. Phys. Lett. 12, 132 (1964)

37. B.S. DeWitt, Dynamical Theory of Groups and Fields (Gordon and Breach, London, 1965) 